\title{
MIXED FINITE ELEMENT APPROXIMATION FOR A COUPLED PETROLEUM RESERVOIR MODEL *
}

\author{
Mohamed Amara $^{1}$, Daniela Capatina-Papaghiuc ${ }^{1}$, Bertrand Denel $^{1}$ \\ AND PEPPINO TERPOLILli ${ }^{2}$
}

\begin{abstract}
In this paper, we are interested in the modelling and the finite element approximation of a petroleum reservoir, in axisymmetric form. The flow in the porous medium is governed by the Darcy-Forchheimer equation coupled with a rather exhaustive energy equation. The semi-discretized problem is put under a mixed variational formulation, whose approximation is achieved by means of conservative Raviart-Thomas elements for the fluxes and of piecewise constant elements for the pressure and the temperature. The discrete problem thus obtained is well-posed and a posteriori error estimates are also established. Numerical tests are presented validating the developed code.
\end{abstract}

Mathematics Subject Classification. 35Q35, 65N15, 65N30, 76S05.

Received: May 18, 2004.

\section{INTRODUCTION}

Due to emerging technologies such as optical fiber sensors, temperature measurements are destined to play a major role in petroleum production logging interpretation. Using temperature recordings from a wellbore and a flowrate history on the surface, it can be envisaged to develop new ways to predict flow repartition among each producing layer of a reservoir or to estimate virgin reservoir temperatures.

In order to solve the inverse problem, one first needs to develop a forward model describing the flow of a monophasic compressible fluid (oil or gas) in a reservoir and a well, from both a dynamic and a thermal point of view. This implies to couple a reservoir model (porous medium) and a well model (based on the compressible Navier-Stokes equations).

In this paper, we only consider the reservoir model, written in axisymmetric form and depending on the cylindrical coordinates $(r, z)$. It consists of the Darcy-Forchheimer equation coupled with a non-standard energy balance (see [10]), notably including the temperature effects due to the decompression of the fluid (JouleThomson effect) and the frictional heating that occurs in the formation. This energy equation thus quantifies the cooling or the heating of the produced fluid before entering the wellbore. Therefore, it stands apart from

Keywords and phrases. Petroleum reservoir, thermometrics, porous medium, mixed finite elements, a posteriori estimators.

* This work is supported by Total under contract DGEP/TDO/CA/RD No.12698.

1 Université de Pau, L.M.A. CNRS-FRE 2570, Avenue de l'Université, 64000 Pau, France. mohamed.amara@univ-pau.fr; daniela.capatina-papaghiuc@univ-pau.fr; bertrand.denel@univ-pau.fr

2 Total, CST Jean Feger, Avenue Larribau, 64018 Pau Cedex, France. peppino.terpolilli@total.com 
the classical models which mainly consider the wellbore thermal exchanges due to conduction and convection and assume that the produced fluid enters the wellbore at the geothermal temperature.

In order to solve this nonlinear system, we first implement a time discretization which uses a classical Euler's scheme and then we linearize the problem at each time step. Its unknowns are now the pressure $p$, the specific flux $\mathbf{G}=\rho \mathbf{v}$, the temperature $T$ and the heat flux $\mathbf{q}=\lambda \nabla T$, the density $\rho$ being updated by verifying the cubic Peng-Robinson state equation at the end of each time loop. The semi-discretized system is shown to be well-posed by means of the Fredholm's alternative, for smooth coefficients and for sufficiently small $\Delta t$.

Concerning the space discretization, we introduce a mixed variational formulation where the convective terms are treated by means of an upwind scheme. To provide an accurate determination of the fluxes, we employ the lowest-order Raviart-Thomas finite elements for the velocity and the heat flux, and piecewise constant finite elements for the pressure and the temperature. We prove that the mixed problem is well-posed thanks to an extension of the Babuška-Brezzi theory ( $c f .[12])$.

Numerical tests are presented, validating the model from both a numerical (convergence in time and space) and a physical (comparison with analytical solutions for the pressure) point of view.

We are next interested in defining and justifying a posteriori estimators, which involve mesh-dependent norms as in [13]. We establish upper and lower error bounds, allowing us to locate the areas where an improvement of the solution is necessary.

An outline of the paper is as follows. In Section 1, we introduce the governing conservation laws and we write the problem in cylindrical coordinates (see also [8]). Once the time discretization and the linearization introduced, Section 2 is devoted to the mathematical study of the semi-discretized problem. The existence and uniqueness of a solution is proved in two steps. Firstly, we neglect the convective terms and we show by means of a non-standard mixed formulation that the obtained problem is well-posed. Moreover, the smoothness of its solution is discussed. Secondly, we show the existence and uniqueness of a solution for the complete problem, by making use of the Fredholm's alternative. The finite element approximation is detailed in the next section. An upwind scheme is introduced and the discrete problem is shown to be well-posed. In Section 4, we present several numerical tests confirming the theoretical results. A comparison with an existing software PIE is given and some realistic cases are also considered on different meshes. Finally, in the last section we define a posteriori error indicators and we carry out some numerical tests which highlight the local character of the estimators.

As a conclusion, we have developed a code for petroleum reservoirs, based on conservative finite elements of Raviart-Thomas and taking into account a non-standard energy equation. In perspective, the mathematical analysis of the error in time is to be done and the code is to be coupled with a wellbore simulator.

\section{Physical modelling}

Our aim is to model the flow of a monophasic compressible fluid (oil or gas) in a petroleum reservoir from both a dynamic and a thermal point of view.

To give an idea of the studied domain, we have represented in Figure 1 a petroleum well, delimited by a casing and surrounded by a cement layer and by the reservoir. The communication between the well and the reservoir is achieved through perforations. The reservoir $\Omega$ is treated as a porous medium divided into several geological layers $\left(\Omega_{i}\right)_{1 \leq i \leq N}$ which are characterized by their own dip and physical properties. Thus, each layer is made of a porous rock (of porosity $\phi$ ), characterized by vertical and horizontal permeabilities, and saturated with both a mobile monophasic fluid (of saturation $s_{o}$ ) and a residual formation water (of saturation $s_{w}$ ).

In what follows, we agree to write the vectors in bold letters and the tensors in underlined bold letters. We shall denote by $c$ any positive constant independent of time and of the space discretization.

\subsection{Conservation laws}

We denote by $\rho$ the density of the fluid, by $\mu$ its viscosity, by $\mathbf{g}=-g \mathbf{e}_{z}$ the gravitational acceleration, by $\phi$ the porosity of the medium and by $\underline{\mathbf{K}}=\left[\begin{array}{cc}k_{1} & 0 \\ 0 & k_{2}\end{array}\right]$ its permeability, with $\phi$ and $\underline{\mathbf{K}}$ depending on the geological layers. We also denote by $\mathbf{v}$ the Darcy velocity and we introduce the specific flux $\mathbf{G}=\rho \mathbf{v}$. 


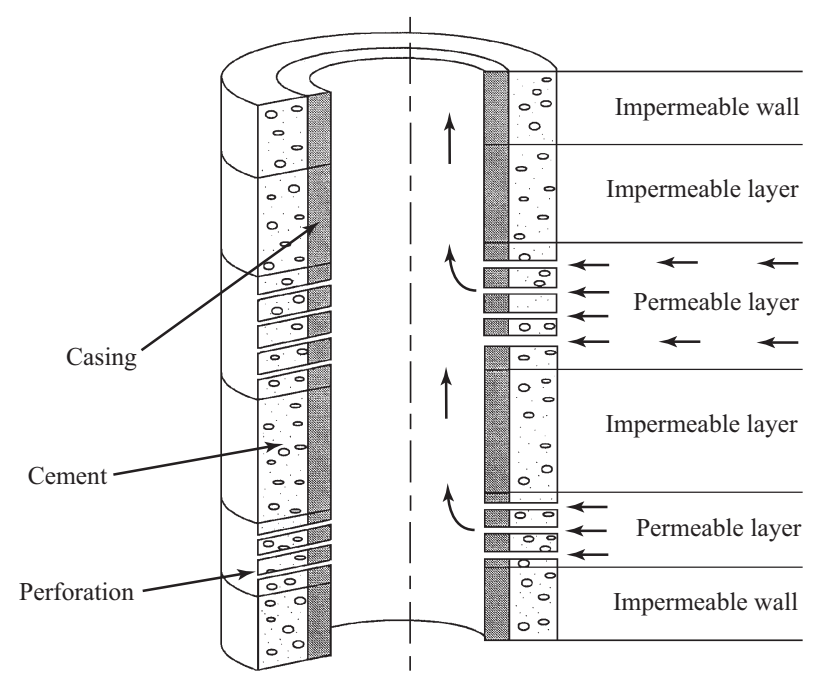

Figure 1. Geometry of a wellbore surrounded by a reservoir.

The fluid flow is modelled by the Darcy-Forchheimer equation coupled with a non-standard energy balance which takes into account, besides the convection and the diffusion, the Joule-Thomson compressibility effect and the frictional heating.

So, the problem is described by the following conservation laws:

$$
\begin{gathered}
\phi \frac{\partial \rho}{\partial t}+\operatorname{div} \mathbf{G}=0, \\
\rho^{-1}\left(\mu \underline{\mathbf{K}}^{-1} \mathbf{G}+F|\mathbf{G}| \mathbf{G}\right)+\nabla p=\rho \mathbf{g}, \\
(\rho c)_{*} \frac{\partial T}{\partial t}+\rho^{-1}(\rho c)_{f} \mathbf{G} \cdot \nabla T-\operatorname{div} \mathbf{q}-\phi \beta T \frac{\partial p}{\partial t}-\rho^{-1}(\beta T-1) \mathbf{G} \cdot \nabla p=0, \\
\rho=\rho(p, T) .
\end{gathered}
$$

Due to the high filtration velocity which can arise around gas wells, we have introduced a quadratic term in the standard Darcy's equation to take into account the kinematic energy losses; $F$ represents the Forchheimer's coefficient.

In the energy equation, $(\rho c)_{*}$ characterizes the heat capacity of a virtual medium, equivalent to the fluid and the porous matrix, while $(\rho c)_{f}$ only symbolizes the fluid properties. The coefficient $\lambda$ denotes the equivalent thermal conductivity, $T$ is the temperature and $\mathbf{q}=\lambda \nabla T$ represents the heat flux.

As usually when modelling petroleum fluids, we use in (4) the Peng-Robinson cubic state equation, see [11].

To this system we add initial conditions for $\rho$ and $T$ :

$$
\rho(x, 0)=\rho_{0}(x) \text { and } T(x, 0)=T_{0}(x) \text { a.e. in } \Omega,
$$

and boundary conditions which will be prescribed later.

\subsection{Problem in cylindrical coordinates}

Due to the geometry of the domain, it is quite natural to write our problem in 2D axisymmetric form, only depending on the cylindrical coordinates $(r, z)$. Following the ideas presented in [8], this means that we consider 


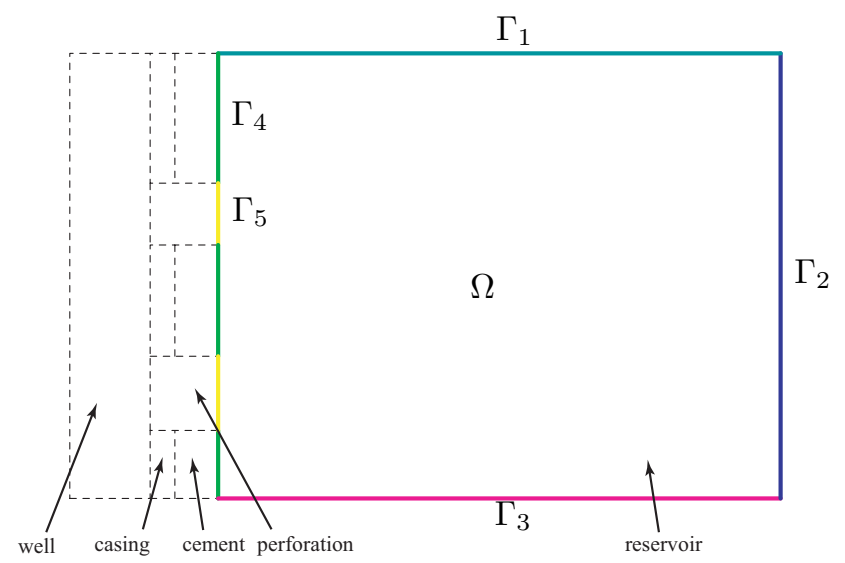

Figure 2. Boundaries of the 2D domain.

a cylindrical reservoir characterized in cartesian, respectively cylindrical coordinates by:

$$
\begin{aligned}
\Omega_{3 D} & =\left\{(x, y, z) ; r_{w}^{2} \leq x^{2}+y^{2} \leq R^{2}, z \in\left[z_{\min }, z_{\max }\right]\right\} \\
& =\left\{(r, \theta, z) ; r_{w} \leq r \leq R, z \in\left[z_{\min }, z_{\max }\right]\right\} .
\end{aligned}
$$

The flow is supposed to be radial and the pressure and the temperature independent of $\theta$.

Thus, our 2D domain merely consists of:

$$
\Omega=\left\{(r, z) ; r_{w} \leq r \leq R, z \in\left[z_{\min }, z_{\max }\right]\right\},
$$

and the $(r, z)$ formulation of our problem is shown to be:

$$
\left\{\begin{array}{l}
r \phi \frac{\partial \rho}{\partial t}+\operatorname{div}(r \mathbf{G})=0 \\
\rho^{-1}\left(\mu \underline{\mathbf{K}}^{-1}+F|\mathbf{G}| \underline{\mathbf{I}}\right) \mathbf{G}+\nabla p=\rho \mathbf{g} \\
\frac{1}{\lambda} \mathbf{q}-\nabla T=0 \\
r(\rho c)_{*} \frac{\partial T}{\partial t}+r \rho^{-1}(\rho c)_{f} \mathbf{G} \cdot \nabla T-\operatorname{div}(r \mathbf{q})-r \phi \beta T \frac{\partial p}{\partial t}-r \rho^{-1}(\beta T-1) \mathbf{G} \cdot \nabla p=0 \\
\rho=\rho(p, T)
\end{array}\right.
$$

where $\mathbf{G}$ now refers to $\left(G_{r}, G_{z}\right)^{t}, \mathbf{q}=\left(q_{r}, q_{z}\right)^{t}$ and $\nabla v=\left(\frac{\partial v}{\partial r}, \frac{\partial v}{\partial z}\right)^{t}$, divv $=\nabla \cdot \mathbf{v}$.

One notes that (5) is a coupled nonlinear system whose unknowns are $\mathbf{G}, \mathbf{q}, p, T$ and $\rho$.

Moreover, all the coefficients related to the porous medium (notably $\underline{\mathbf{K}}$ and $\lambda$ ) are discontinuous across the interfaces of the geological layers.

\subsection{Boundary conditions}

In order to define the boundary conditions, $\Gamma=\partial \Omega$ is divided into five parts, see Figure 2 .

The boundary conditions apply to $\mathbf{G}$ and its dual variable $p$, as well as to $\mathbf{q}$ and $T$.

Concerning the specific flux, a condition of impermeability $\mathbf{G} \cdot \mathbf{n}=0$ is imposed on $\Gamma_{1}, \Gamma_{3}$ and $\Gamma_{4}$. On the external boundary $\Gamma_{2}$, either a normal specific flux or a pressure $p=p_{\Gamma}$ can be set. This most notably allows us 
to treat the standard cases of a closed reservoir (no-flow condition $\mathbf{G} \cdot \mathbf{n}=0$ ) and of a reservoir feed at constant pressure. It goes the same way for the perforations located on $\Gamma_{5}$.

Concerning the temperature, the geothermal gradient is imposed on the top $\Gamma_{1}$ and the bottom $\Gamma_{3}$ of the reservoir, whereas a null normal flux condition or a temperature $T_{\Gamma}$ can be set on the lateral boundaries $\Gamma_{2}, \Gamma_{4}$ and $\Gamma_{5}$.

From now on, we denote by $\Gamma_{p}, \Gamma_{T}, \Gamma_{G}$ and $\Gamma_{q}$ respectively the union of the boundaries where a pressure $p_{\Gamma}$, a temperature $T_{\Gamma}$, a normal specific flux $Q$ and a normal heat flux $\Psi$ are imposed. So, we have $\bar{\Gamma}=\bar{\Gamma}_{G} \cup \bar{\Gamma}_{p}=$ $\bar{\Gamma}_{q} \cup \bar{\Gamma}_{T}$. In order to simplify the presentation, we assume in what follows that $\Gamma_{p} \neq \varnothing$ and $\Gamma_{T} \neq \varnothing$.

\section{AnAlysis OF THE SEMI-DISCRETIZED PROBLEM}

\subsection{Time discretization}

The time discretization is based on the classical Euler's implicit scheme. At any time increment, we determine the unknowns $\mathbf{G}, \mathbf{q}, p$ and $T$, and then we update $\rho$ by satisfying the Peng-Robinson cubic equation. This last step is achieved by means of a thermodynamic module.

With this aim in view, let us first replace in the mass conservation equation the derivatives of $\rho$ thanks to its dependency in $p$ and $T$ :

$$
\frac{\partial \rho}{\partial t}=\chi \rho \frac{\partial p}{\partial t}-\beta \rho \frac{\partial T}{\partial t}
$$

where we have introduced the compressibility coefficient $\chi$, respectively the expansion coefficient $\beta$ by putting:

$$
\chi=\frac{1}{\rho}\left(\frac{\partial \rho}{\partial p}\right)_{T}, \beta=\frac{1}{V}\left(\frac{\partial V}{\partial T}\right)_{p}=-\frac{1}{\rho}\left(\frac{\partial \rho}{\partial T}\right)_{p} .
$$

This leads to the following time-discretized linear problem:

$$
\left\{\begin{array}{l}
\frac{1}{\rho^{n-1}}\left(\mu^{n-1} \underline{\mathbf{K}}^{-1}+F\left|\mathbf{G}^{n-1}\right| \underline{\mathbf{I}}\right) \mathbf{G}^{n}+\nabla p^{n}=\rho^{n-1} \mathbf{g}, \\
\frac{1}{\lambda^{n-1}} \mathbf{q}^{n}-\nabla T^{n}=0 \\
r \frac{\phi \rho^{n-1} \chi^{n-1}}{\Delta t} p^{n}-r \frac{\phi \rho^{n-1} \beta^{n-1}}{\Delta t} T^{n}+\operatorname{div}\left(r \mathbf{G}^{n}\right)=r \frac{\phi \rho^{n-1} \chi^{n-1}}{\Delta t} p^{n-1}-r \frac{\phi \rho^{n-1} \beta^{n-1}}{\Delta t} T^{n-1} \\
r \frac{(\rho c)_{*}^{n-1}}{\Delta t} T^{n}+r \frac{(\rho c)_{f}^{n-1}}{\rho^{n-1}} \mathbf{G}^{n-1} \cdot \nabla T^{n}-r \frac{\phi \beta^{n-1} T^{n-1}}{\Delta t} p^{n}, \\
\quad-r \frac{\left(\beta^{n-1} T^{n-1}-1\right)}{\rho^{n-1}} \mathbf{G}^{n-1} \cdot \nabla p^{n}-\operatorname{div}\left(r \mathbf{q}^{n}\right)=r \frac{(\rho c)_{*}^{n-1}}{\Delta t} T^{n-1}-r \frac{\phi \beta^{n-1} T^{n-1}}{\Delta t} p^{n-1}
\end{array}\right.
$$

For the sake of clarity, let us introduce some notations for the thermodynamic coefficients:

$$
\begin{gathered}
a=\phi \rho^{n-1} \chi^{n-1}, b=\phi \rho^{n-1} \beta^{n-1}, d=(\rho c)_{*}^{n-1}, \\
f=\phi \beta^{n-1} T^{n-1}, k=\frac{(\rho c)_{f}^{n-1}}{\rho^{n-1}}, l=\frac{1-\beta^{n-1} T^{n-1}}{\rho^{n-1}},
\end{gathered}
$$

and let us also introduce the symmetric tensor:

$$
\underline{\mathbf{M}}=\frac{1}{\rho^{n-1}}\left(\mu^{n-1} \underline{\mathbf{K}}^{-1}+\frac{F}{r}\left|\mathbf{G}^{n-1}\right| \underline{\mathbf{I}}\right) .
$$

One can notice that $a, b, d, f, k, \lambda$ and $\underline{\mathbf{M}}$ are positive, respectively positive definite, whereas $l$ is of variable sign. 
We agree to make the change of variables $\widetilde{\mathbf{G}}=r \mathbf{G}, \widetilde{\mathbf{q}}=r \mathbf{q}$ and to denote from now on $\widetilde{\mathbf{G}}, \widetilde{\mathbf{q}}$ by $\mathbf{G}, \mathbf{q}$. Moreover, for the simplicity of the presentation, we drop the index $n-1$ on the coefficients of the problem.

We are now interested in the mathematical analysis of the previous linearized problem, which writes as follows:

$$
\left\{\begin{array}{l}
\frac{1}{r} \underline{\mathbf{M}} \mathbf{G}+\nabla p=\rho^{n-1} \mathbf{g}, \\
\frac{1}{r \lambda} \mathbf{q}-\nabla T=0, \\
r \frac{a}{\Delta t} p-r \frac{b}{\Delta t} T+\operatorname{div} \mathbf{G}=r \frac{a}{\Delta t} p^{n-1}-r \frac{b}{\Delta t} T^{n-1}, \\
r \frac{d}{\Delta t} T+k \mathbf{G}^{n-1} \cdot \nabla T-r \frac{f}{\Delta t} p+l \mathbf{G}^{n-1} \cdot \nabla p-\operatorname{div} \mathbf{q}=r \frac{d}{\Delta t} T^{n-1}-r \frac{f}{\Delta t} p^{n-1} .
\end{array}\right.
$$

The unknowns $\mathbf{G}, \mathbf{q}, p$ and $T$ satisfy the boundary conditions:

$$
\mathbf{G} \cdot \mathbf{n}=Q \text { on } \Gamma_{G}, \mathbf{q} \cdot \mathbf{n}=\Psi \text { on } \Gamma_{q}, p=p_{\Gamma} \text { on } \Gamma_{p} \text { and } T=T_{\Gamma} \text { on } \Gamma_{T},
$$

as well as classical transmission conditions at the interfaces between the geological layers $\Omega_{i}, i=1, \ldots, N$. Let us recall that $\underline{\mathbf{M}}$ and $\lambda$ are discontinuous across the interfaces of $\Omega_{i}, i=1, \ldots, N$.

From now on, we make the following assumptions on the thermodynamic coefficients, which are justified in practice by all the available experimental data:

(A1) $a, d, \frac{1}{\lambda}$ are bounded from below by a strictly positive constant and $\underline{\mathbf{M}}$ is uniformly positive definite;

(A2) $a, b, \hat{d}, f, k, l, \frac{1}{\lambda}$ and $\underline{\mathbf{M}}$ are bounded a.e. in $\Omega$;

(A3) $\exists c \in \mathbb{R}_{+}^{*}$ such that $4 a d-(b+f)^{2} \geq c$ a.e. in $\Omega$.

\subsection{Well-posedness of the problem without convection}

Let us begin the mathematical analysis by neglecting, for the moment, the convective terms $k \mathbf{G}^{n-1} \cdot \nabla T$ and $l \mathbf{G}^{n-1} \cdot \nabla p$ in the energy equation and by writing next the problem (7) under variational form.

We denote by $\mathbf{V}=(\mathbf{G}, \mathbf{q})$ the vector unknowns, respectively by $s=(p, T)$ the scalar ones and we introduce the spaces:

$$
\begin{gathered}
\mathbb{L}^{2}(\Omega)=L^{2}(\Omega) \times L^{2}(\Omega), \\
\mathbb{H}(\operatorname{div}, \Omega)=H(\operatorname{div}, \Omega) \times H(\operatorname{div}, \Omega), \\
\mathbb{H}^{0}(\operatorname{div}, \Omega)=\left\{\mathbf{V}^{\prime}=\left(\mathbf{G}^{\prime}, \mathbf{q}^{\prime}\right) \in \mathbb{H}(\operatorname{div}, \Omega) ; \mathbf{G}^{\prime} \cdot \mathbf{n}=0 \text { on } \Gamma_{G}, \mathbf{q}^{\prime} \cdot \mathbf{n}=0 \text { on } \Gamma_{q}\right\}, \\
\mathbb{H}^{*}(\operatorname{div}, \Omega)=\left\{\mathbf{V}^{\prime}=\left(\mathbf{G}^{\prime}, \mathbf{q}^{\prime}\right) \in \mathbb{H}(\operatorname{div}, \Omega) ; \mathbf{G}^{\prime} \cdot \mathbf{n}=Q \text { on } \Gamma_{G}, \mathbf{q}^{\prime} \cdot \mathbf{n}=\Psi \text { on } \Gamma_{q}\right\},
\end{gathered}
$$

endowed with their natural norms $\|\cdot\|_{0, \Omega}$ and $|\|\cdot \mid\|$.

By multiplying the equations of (7) by test-functions $\left(\mathbf{V}^{\prime}, s^{\prime}\right)$ and after integrating by parts, one gets the following mixed problem:

$$
\left\{\begin{array}{l}
\text { Find } \mathbf{V} \in \mathbb{H}^{*}(\operatorname{div}, \Omega), s \in \mathbb{L}^{2}(\Omega) \text { such that } \\
A\left(\mathbf{V}, \mathbf{V}^{\prime}\right)+B\left(s, \mathbf{V}^{\prime}\right)=F_{1}\left(\mathbf{V}^{\prime}\right) \quad \forall \mathbf{V}^{\prime} \in \mathbb{H}^{0}(\operatorname{div}, \Omega), \\
-B\left(s^{\prime}, \mathbf{V}\right)+C\left(s, s^{\prime}\right)=F_{2}\left(s^{\prime}\right) \quad \forall s^{\prime} \in \mathbb{L}^{2}(\Omega),
\end{array}\right.
$$


where the bilinear forms are defined by

$$
\begin{aligned}
& A\left(\mathbf{V}, \mathbf{V}^{\prime}\right)=\int_{\Omega} \frac{1}{r} \underline{\mathbf{M G}} \cdot \mathbf{G}^{\prime} \mathrm{dx}+\int_{\Omega} \frac{1}{r \lambda} \mathbf{q} \cdot \mathbf{q}^{\prime} \mathrm{dx} \\
& B\left(s, \mathbf{V}^{\prime}\right)=-\int_{\Omega} p \operatorname{div} \mathbf{G}^{\prime} \mathrm{dx}+\int_{\Omega} T \operatorname{div} \mathbf{q}^{\prime} \mathrm{dx} \\
& C\left(s, s^{\prime}\right)=\int_{\Omega} r \frac{a}{\Delta t} p p^{\prime} \mathrm{dx}-\int_{\Omega} r \frac{b}{\Delta t} T p^{\prime} \mathrm{dx}+\int_{\Omega} r \frac{d}{\Delta t} T T^{\prime} \mathrm{dx}-\int_{\Omega} r \frac{f}{\Delta t} p T^{\prime} \mathrm{dx},
\end{aligned}
$$

and the linear forms by

$$
\begin{aligned}
& F_{1}\left(\mathbf{V}^{\prime}\right)=\int_{\Omega} \rho^{n-1} \mathbf{g} \cdot \mathbf{G}^{\prime} \mathrm{dx}-\left\langle\mathbf{G}^{\prime} \cdot \mathbf{n}, p_{\Gamma}\right\rangle_{\Gamma}+\left\langle\mathbf{q}^{\prime} \cdot \mathbf{n}, T_{\Gamma}\right\rangle_{\Gamma} \\
& F_{2}\left(s^{\prime}\right)=\int_{\Omega} \frac{r}{\Delta t}\left(a p^{n-1}-b T^{n-1}\right) p^{\prime} \mathrm{dx}+\int_{\Omega} \frac{r}{\Delta t}\left(d T^{n-1}-f p^{n-1}\right) T^{\prime} \mathrm{dx} .
\end{aligned}
$$

Here above, we employed the notation $\langle\cdot, \cdot\rangle_{\Gamma}$ for the duality product between $H^{-1 / 2}(\Gamma)$ and $H^{1 / 2}(\Gamma)$. Then one can establish:

Theorem 2.1. Assume that $\rho^{n-1}, p^{n-1}, T^{n-1} \in L^{2}(\Omega)$ and that the coefficients $a, b, d, f, \lambda$ and $\underline{M}$ satisfy the hypotheses (A1) to (A3). Then the mixed problem (8) has a unique solution.

Proof. Thanks to $(A 1)$ and $(A 2)$, the forms $A(\cdot, \cdot), B(\cdot, \cdot), C(\cdot, \cdot)$ and $F_{1}(\cdot), F_{2}(\cdot)$ are obviously continuous and moreover,

$$
A\left(\mathbf{V}^{\prime}, \mathbf{V}^{\prime}\right) \geq c\left\|\mathbf{V}^{\prime}\right\|_{0, \Omega}^{2}, \forall \mathbf{V}^{\prime} \in \mathbb{H}^{0}(\operatorname{div}, \Omega)
$$

since $\frac{1}{r} \geq \frac{1}{r_{w}}$. So $A(\cdot, \cdot)$ is $\mathbb{H}(\operatorname{div}, \Omega)$-elliptic on

$$
\operatorname{Ker} B=\left\{\mathbf{V}^{\prime}=\left(\mathbf{G}^{\prime}, \mathbf{q}^{\prime}\right) \in \mathbb{H}^{0}(\operatorname{div}, \Omega) ; \operatorname{div} \mathbf{G}^{\prime}=\operatorname{div} \mathbf{q}^{\prime}=0 \text { in } \Omega\right\}
$$

One can easily show that $B(\cdot, \cdot)$ satisfies the inf-sup condition.

Indeed, with any $s=(p, T) \in \mathbb{L}^{2}(\Omega)$ one associates $\mathbf{G}^{\prime}=\nabla \varsigma, \mathbf{q}^{\prime}=\nabla \phi$ where $\varsigma, \phi$ are the unique solutions of the following boundary value problems:

$$
\left\{\begin{array}{l}
-\Delta \varsigma=p \text { in } \Omega \\
\varsigma=0 \text { on } \Gamma_{p} \\
\frac{\partial \varsigma}{\partial n}=0 \text { on } \Gamma_{G}
\end{array} ; \quad\left\{\begin{array}{l}
-\Delta \phi=T \text { in } \Omega \\
\phi=0 \text { on } \Gamma_{T} \\
\frac{\partial \phi}{\partial n}=0 \text { on } \Gamma_{q}
\end{array} .\right.\right.
$$

Thus, the couple $\mathbf{V}^{\prime}=\left(\mathbf{G}^{\prime}, \mathbf{q}^{\prime}\right)$ belongs to $\mathbb{H}^{0}(\operatorname{div}, \Omega)$ and one has

$$
\forall s \in \mathbb{L}^{2}(\Omega), \sup _{\mathbf{V} \in \mathbb{H}^{0}(\operatorname{div}, \Omega)} \frac{B(s, \mathbf{V})}{\|\mid \mathbf{V}\|} \geq \frac{B\left(s, \mathbf{V}^{\prime}\right)}{\left\|\mathbf{V}^{\prime}\right\|} \geq c\|s\|_{0, \Omega}
$$

where $c$ depends only on the domain $\Omega$.

In addition to the inf-sup condition for $B(\cdot, \cdot)$ and the ellipticity of $A(\cdot, \cdot)$ on $\operatorname{Ker} B$, one has that $C(\cdot, \cdot)$ is positive definite. 
Indeed, thanks to $(A 3)$, one has that

$$
\begin{aligned}
C(s, s) & =\int_{\Omega} \frac{r}{\Delta t}\left[a p^{2}+d T^{2}-(b+f) T p\right] \mathrm{dx} \\
& =\int_{\Omega} \frac{r}{\Delta t}\left[\left(\sqrt{a} p-\frac{b+f}{2 \sqrt{a}} T\right)^{2}+\frac{4 a d-(b+f)^{2}}{4 a} T^{2}\right] \mathrm{dx} \\
& \geq \frac{c}{\Delta t}\left(\|p\|_{0, \Omega}^{2}+\|T\|_{0, \Omega}^{2}\right) .
\end{aligned}
$$

Therefore, the relation $A(\mathbf{V}, \mathbf{V})+C(s, s)=0$ implies that $\mathbf{V}=\mathbf{0}, s=0$, which ensures the uniqueness of the solution of problem (8).

In order to prove the existence of a solution, we use a Galerkin method. First of all, let us notice that the classical Babuška-Brezzi theorem gives the well-posedness of problem $(8)$ with $C(\cdot, \cdot)=0$, which allows us to consider next $F_{1}(\cdot)=0$. So, we solve the problem on a sequence of finite dimensional spaces $\mathbb{H}_{m} \times \mathbb{L}_{m}$, spanned by the first $m$ vectors of a Hilbert basis in $\mathbb{H}(\operatorname{div}, \Omega) \times \mathbb{L}^{2}(\Omega)$.

The unique solution $\left(\mathbf{V}_{m}, s_{m}\right) \in \mathbb{H}_{m} \times \mathbb{L}_{m}$ satisfies $A\left(\mathbf{V}_{m}, \mathbf{V}_{m}\right)+C\left(s_{m}, s_{m}\right)=F_{2}\left(s_{m}\right)$.

The positivity of $A(\cdot, \cdot)$ and the coercivity of $C(\cdot, \cdot)$ together with the inf-sup condition on $B(\cdot, \cdot)$, give that both $\left(s_{m}\right)$ and $\left(\mathbf{V}_{m}\right)$ are bounded with respect to $m$. Passing to the limit when $m \rightarrow \infty$, one classically obtains the existence of a solution. We have thus established that the variational problem (8) is well-posed.

Remark 2.2. The bilinear form $C(\cdot, \cdot)$ being non-symmetric, one cannot use the results of Brezzi and Fortin [5] in order to prove the well-posedness of problem (8).

In order to write our problem by means of a linear continuous operator, let us first denote the data of the initial problem (7) by:

where

$$
\mathbf{f}=\left(f_{\Omega}, f_{\Gamma}\right) \in \mathbb{X}_{1} \times \mathbb{X}_{2},
$$

$$
\begin{aligned}
& f_{\Omega}=\left(\mathbf{f}_{1}, \mathbf{f}_{2}, f_{3}, f_{4}\right) \in \mathbb{X}_{1}=\mathbb{L}^{2}(\Omega) \times \mathbb{L}^{2}(\Omega) \times L^{2}(\Omega) \times L^{2}(\Omega), \\
& f_{\Gamma}=\left(p_{\Gamma}, T_{\Gamma}, Q, \Psi\right) \in \mathbb{X}_{2}=H^{1 / 2}\left(\Gamma_{p}\right) \times H^{1 / 2}\left(\Gamma_{T}\right) \times H^{-1 / 2}\left(\Gamma_{G}\right) \times H^{-1 / 2}\left(\Gamma_{q}\right),
\end{aligned}
$$

with, in our case,

$$
\mathbf{f}_{1}=\rho^{n-1} \mathbf{g}, \mathbf{f}_{2}=\mathbf{0}, f_{3}=\frac{r}{\Delta t}\left(a p^{n-1}-b T^{n-1}\right) \text { and } f_{4}=\frac{r}{\Delta t}\left(d T^{n-1}-f p^{n-1}\right) .
$$

With these notations, the right-hand side term of problem (8) can be written under the following form:

$$
\begin{aligned}
& F_{1}\left(\mathbf{V}^{\prime}\right)=\int_{\Omega} \mathbf{f}_{1} \cdot \mathbf{G}^{\prime} \mathrm{dx}+\int_{\Omega} \mathbf{f}_{2} \cdot \mathbf{q}^{\prime} \mathrm{dx}-\left\langle\mathbf{G}^{\prime} \cdot \mathbf{n}, p_{\Gamma}\right\rangle_{\Gamma}+\left\langle\mathbf{q}^{\prime} \cdot \mathbf{n}, T_{\Gamma}\right\rangle_{\Gamma}, \\
& F_{2}\left(s^{\prime}\right)=\int_{\Omega} f_{3} p^{\prime} \mathrm{dx}+\int_{\Omega} f_{4} T^{\prime} \mathrm{dx} .
\end{aligned}
$$

Then, thanks to Theorem 2.1, we can define a linear continuous operator

$$
\mathcal{L}: \mathbb{X}_{1} \times \mathbb{X}_{2} \longrightarrow \mathbb{H}(\operatorname{div}, \Omega) \times \mathbb{L}^{2}(\Omega),
$$

which associates, with any data $\mathbf{f}$, the unique solution of (8):

$$
\sigma=(\mathbf{V}, s) \in \mathbb{H}(\operatorname{div}, \Omega) \times \mathbb{L}^{2}(\Omega) .
$$

Finally, the variational problem is equivalent to $\mathcal{L} \mathbf{f}=\sigma$. 


\subsection{Smoothness of the solution}

We show here that for sufficiently smooth boundary conditions and thermodynamic coefficients, the solution of (8) is smoother on each geological layer $\Omega_{i}, i=1, \ldots, N$. More precisely, we shall prove in the next theorem that the previous operator $\mathcal{L}$ is well-defined from $\mathbb{X}_{1} \times \mathbb{X}_{3}$ to $\mathbb{H}(\operatorname{div}, \Omega) \times \mathbb{Y}$, with

$$
\begin{gathered}
\mathbb{X}_{3}=\prod_{i=1}^{N}\left[H^{1 / 2+\delta}\left(\Gamma_{p}^{i}\right) \times H^{1 / 2+\delta}\left(\Gamma_{T}^{i}\right) \times H^{-1 / 2+\delta}\left(\Gamma_{G}^{i}\right) \times H^{-1 / 2+\delta}\left(\Gamma_{q}^{i}\right)\right] \\
\mathbb{Y}=\prod_{i=1}^{N} \mathbb{H}^{1+\delta}\left(\Omega_{i}\right),
\end{gathered}
$$$$
\text { and } 0<\delta \leq 1
$$

We have put:

$$
\begin{gathered}
H^{1 / 2+\delta}\left(\Gamma_{p}^{i}\right)=H^{1 / 2+\delta}\left(\Gamma_{p} \cap \partial \Omega_{i}\right), \quad H^{1 / 2+\delta}\left(\Gamma_{T}^{i}\right)=H^{1 / 2+\delta}\left(\Gamma_{T} \cap \partial \Omega_{i}\right), \\
H^{-1 / 2+\delta}\left(\Gamma_{G}^{i}\right)=H^{-1 / 2+\delta}\left(\Gamma_{G} \cap \partial \Omega_{i}\right), \quad H^{-1 / 2+\delta}\left(\Gamma_{q}^{i}\right)=H^{-1 / 2+\delta}\left(\Gamma_{q} \cap \partial \Omega_{i}\right) .
\end{gathered}
$$

We suppose next that, at any $t^{n}$, there exists a lifting $\left(\mathbf{V}^{*}, s^{*}\right) \in \mathbb{H}(\operatorname{div}, \Omega) \times \mathbb{Y}$ satisfying the imposed boundary conditions.

We can then establish:

Theorem 2.3. Suppose that $\rho^{n-1} \in H^{1}(\Omega), \nabla \lambda \in L^{\infty}\left(\Omega_{i}\right)$ and $\underline{\boldsymbol{M}}^{-1}=\left(m_{k l}\right)_{1 \leq k, l \leq 2} \in \mathcal{C}^{0,1}\left(\overline{\Omega_{i}}\right)$, where $m_{k l}$ are Lipschitz functions for each $i=1, \ldots, N$.

Then, for any $\left(p_{\Gamma}, T_{\Gamma}, Q, \Psi\right) \in \mathbb{X}_{3}$, one gets that $s \in \mathbb{Y}$, where $\sigma=(\boldsymbol{V}, s)$ is the unique solution of (8).

Proof. First of all, an integration by parts on each subdomain $\Omega_{i}$ gives that:

$$
B\left(s, \mathbf{V}^{\prime}\right)=\sum_{i=1}^{N}\left(\int_{\Omega_{i}} \nabla p \cdot \mathbf{G}^{\prime} \mathrm{dx}-\int_{\Omega_{i}} \nabla T \cdot \mathbf{q}^{\prime} \mathrm{d} \mathbf{x}\right)-\sum_{i=1}^{N} \int_{\partial \Omega_{i}} p \mathbf{G}^{\prime} \cdot \mathbf{n} \mathrm{d} \sigma+\sum_{i=1}^{N} \int_{\partial \Omega_{i}} T \mathbf{q}^{\prime} \cdot \mathbf{n} \mathrm{d} \sigma .
$$

By taking $\mathbf{V}^{\prime} \in \mathcal{D}\left(\Omega_{i}\right)$ as test-function in the first variational equation of (8), one gets that:

$$
\nabla T=\frac{1}{\lambda r} \mathbf{q}, \quad \nabla p=\rho^{n-1} \mathbf{g}-\frac{1}{r} \underline{\mathbf{M G}} \text { in } \Omega_{i}, \quad i=1, \ldots, N .
$$

Next, since $\mathbf{G}^{\prime} \cdot \mathbf{n}$ and $\mathbf{q}^{\prime} \cdot \mathbf{n}$ are continuous across the interfaces of the subdomains, it comes that $p$ and $T$ are also continuous across the interfaces. Finally, this implies that $p, T \in H^{1}(\Omega)$.

One can now write in each layer $\Omega_{i}$ that:

$$
\left\{\begin{array}{l}
\operatorname{div}\left(\frac{1}{r} \mathbf{q}\right)=\nabla \lambda \cdot \nabla T+\lambda \Delta T \\
\operatorname{div}\left(\frac{1}{r} \mathbf{G}\right)=-\operatorname{div}\left(\rho^{n-1} \underline{\mathbf{M}}^{-1} \mathbf{g}\right)-\operatorname{div}\left(\underline{\mathbf{M}}^{-1} \nabla p\right)
\end{array}\right.
$$

Since $r$ is bounded and since $\operatorname{divq}$, $\operatorname{div} \mathbf{G} \in L^{2}(\Omega)$, it comes that:

$$
\Delta T \in L^{2}\left(\Omega_{i}\right), \quad \operatorname{div}\left(\underline{\mathbf{M}}^{-1} \nabla p\right) \in L^{2}\left(\Omega_{i}\right) .
$$


Let us also notice that

$$
T=T_{\Gamma} \in H^{1 / 2+\delta}\left(\Gamma_{T}^{i}\right), \quad \lambda \nabla T \cdot \mathbf{n}=\frac{1}{r} \mathbf{q} \cdot \mathbf{n} \in H^{-1 / 2+\delta}\left(\Gamma_{q}^{i}\right)
$$

and similarly

$$
p=p_{\Gamma} \in H^{1 / 2+\delta}\left(\Gamma_{p}^{i}\right), \quad \underline{\mathbf{M}}^{-1} \nabla p \cdot \mathbf{n}=-\rho^{n-1} \underline{\mathbf{M}}^{-1} \mathbf{g} \cdot \mathbf{n}-\frac{1}{r} \mathbf{G} \cdot \mathbf{n} \in H^{-1 / 2+\delta}\left(\Gamma_{G}^{i}\right) .
$$

Moreover, the following transmission conditions hold at the interfaces between the subdomains:

$$
[T]=0,[p]=0,[\lambda \nabla T \cdot \mathbf{n}]=0 \text { and }\left[\underline{\mathbf{M}}^{-1} \nabla p \cdot \mathbf{n}\right]=0 .
$$

The existence of the above mentioned lifting ensures that the Dirichlet and Neumann boundary data satisfy usual compatibility conditions.

Then, thanks to the regularity of an elliptic problem with discontinuous coefficients on a polygon (see for instance Grisvard [9]), it finally comes that

$$
(p, T) \in \prod_{i=1}^{N} \mathbb{H}^{1+\delta}\left(\Omega_{i}\right)
$$

which ends the proof.

Remark 2.4. One cannot have $(p, T) \in \mathbb{H}^{2}(\Omega)$, due to the discontinuity of $\lambda$ and $\underline{\mathbf{M}}$ at the interfaces.

Remark 2.5. Whenever the boundary conditions are sufficiently smooth and each layer $\Omega_{i}$ is a convex domain, one gets $s=1$.

Remark 2.6. Let us recall that $\nabla \rho=\chi \rho \nabla p-\beta \rho \nabla T$ and, since $p$ and $T$ are shown to be in $H^{1}(\Omega)$ with no additional condition, the hypothesis $\rho^{n-1} \in H^{1}(\Omega)$ is quite natural.

An important point in theorem 2.3 is that one conserves the regularity of the solution from one time step to another, for sufficiently smooth initial boundary conditions.

Indeed, if the boundary conditions at $t^{n-1}$ belong to $\mathbb{X}_{3}$, then according to the proof of Theorem 2.3 it comes that $p^{n} \in H^{1 / 2+\delta}\left(\Gamma_{p}^{i}\right)$ and $T^{n} \in H^{1 / 2+\delta}\left(\Gamma_{T}^{i}\right), i=1, \ldots, N$. Moreover,

$$
\mathbf{G}^{n}=-r \rho^{n-1} \underline{\mathbf{M}}^{-1} \mathbf{g}-r \underline{\mathbf{M}}^{-1} \nabla p^{n} \in \prod_{i=1}^{N} \mathbb{H}^{\delta}\left(\Omega_{i}\right) \text { and } \mathbf{q}^{n}=r \lambda \nabla T^{n} \in \prod_{i=1}^{N} \mathbb{H}^{\delta}\left(\Omega_{i}\right),
$$

which implies that:

$$
\mathbf{G}^{n} \cdot \mathbf{n} \in H^{-1 / 2+\delta}\left(\Gamma_{G}^{i}\right), \mathbf{q}^{n} \cdot \mathbf{n} \in H^{-1 / 2+\delta}\left(\Gamma_{q}^{i}\right)
$$

So the boundary conditions at $t^{n}$ are also in $\mathbb{X}_{3}$.

\subsection{Fredholm's alternative for the problem with convection}

Let us now take into account the convective terms and define therefore the linear continuous operator

$$
K: \mathbb{Y} \longrightarrow L^{2}(\Omega), \quad K(s)=k \mathbf{G}^{n-1} \cdot \nabla T+l \mathbf{G}^{n-1} \cdot \nabla p .
$$

The main point is that the operator $K$ is compact thanks to the compact embedding $H^{\delta}\left(\Omega_{i}\right) \hookrightarrow L^{2}\left(\Omega_{i}\right)$, for $i=1, \ldots, N$ and $0<\delta \leq 1$. 
We also need to introduce $\mathcal{K}: \mathbb{H}(\operatorname{div}, \Omega) \times \mathbb{Y} \longrightarrow \mathbb{X}_{1} \times \mathbb{X}_{3}$,

$$
\mathcal{K}(\sigma)=\left(f_{\Omega}, f_{\Gamma}\right)
$$

with $f_{\Omega}=(\mathbf{0}, \mathbf{0}, 0, K(s))$ and $f_{\Gamma}=\mathbf{0}$.

Then our initial problem (7) can be put under the following form:

$$
\sigma=\mathcal{L}(\mathbf{f}+\mathcal{K}(\sigma)) \Longleftrightarrow(\mathcal{I}-\mathcal{L} \mathcal{K}) \sigma=\mathcal{L} \mathbf{f}
$$

where $\mathcal{L} \mathcal{K}$ is now a compact operator from $\mathbb{H}(\operatorname{div}, \Omega) \times \mathbb{Y}$ to itself.

In order to prove the well-posedness of problem (10), we apply the Fredholm's theory. More precisely, we establish next that $\operatorname{Ker}(\mathcal{I}-\mathcal{L K})=\{0\}$, therefore problem (10) has a unique solution for any right-hand side term.

Lemma 2.7. Under assumptions $(A 1),(A 2),(A 3)$ and for $\Delta t$ sufficiently small, one has $\operatorname{Ker}(\mathcal{I}-\mathcal{L} \mathcal{K})=\{0\}$.

Proof. The solution of the equation $(\mathcal{I}-\mathcal{L} \mathcal{K}) \sigma=0$ satisfies the following relations, obtained by taking $\tau=\sigma$ as test-function in the variational formulation:

$$
\left\{\begin{array}{l}
A(\mathbf{V}, \mathbf{V})+B(s, \mathbf{V})=0 \\
-B(s, \mathbf{V})+C(s, s)+\int_{\Omega} K(s) T \mathrm{dx}=0
\end{array}\right.
$$

By adding these equalities, it comes that

$$
A(\mathbf{V}, \mathbf{V})+C(s, s)+\int_{\Omega} K(s) T \mathrm{dx}=0
$$

By replacing $\nabla T=\frac{1}{r \lambda} \mathbf{q}, \nabla p=-\frac{1}{r} \underline{\mathbf{M G}}$ and by means of the Gauss reduction, the previous relation can be finally written as below:

$$
\begin{aligned}
\int_{\Omega} \frac{1}{r} \underline{\mathbf{M}}\left(\mathbf{G}-\frac{l}{2} T \mathbf{G}^{n-1}\right) \cdot\left(\mathbf{G}-\frac{l}{2} T \mathbf{G}^{n-1}\right) \mathrm{dx} & +\int_{\Omega} \frac{1}{r \lambda}\left|\mathbf{q}+\frac{k}{2} T \mathbf{G}^{n-1}\right|^{2} \mathrm{dx}+\int_{\Omega} \frac{r}{\Delta t}\left(\sqrt{a} p-\frac{b+f}{2 \sqrt{a}} T\right)^{2} \mathrm{dx} \\
& +\int_{\Omega} \frac{r}{4 \Delta t}\left(\frac{4 a d-(b+f)^{2}}{a}-\frac{\Delta t}{r^{2}} \underline{\mathbf{M}} \mathbf{G}^{n-1} \cdot \mathbf{G}^{n-1}\right) T^{2} \mathrm{dx}=0
\end{aligned}
$$

with $\underline{\widetilde{\mathbf{M}}}=l^{2} \underline{\mathbf{M}}+\frac{k^{2}}{\lambda} \underline{\mathbf{I}}$ positive definite and with $4 a d-(b+f)^{2} \geq c>0$ due to $(A 3)$.

Then, for

$$
\Delta t<r^{2} \frac{4 a d-(b+f)^{2}}{a \underline{\mathbf{M}} \mathbf{G}^{n-1} \cdot \mathbf{G}^{n-1}},
$$

one gets that all the squares vanish, which implies that $\sigma=0$.

As a conclusion, we have established in this section the well-posedness of the time-discretized problem (7) at any $t^{n}$, under nonrestrictive regularity assumptions on the data but for a sufficiently small time step. 


\section{Finite element approximation}

\subsection{The discrete problem with upwinding}

We are now interested in the space discretization of problem (7), which will be achieved by means of low-order conforming finite elements.

Let us consider a family $\left(\mathcal{T}_{h}\right)_{h}$ of triangulations of $\bar{\Omega}$ consisting of triangles matching at the interfaces between the layers $\Omega_{i}$, and with $\left(\mathcal{T}_{h}\right)_{h}$ regular in the sense of Ciarlet [7].

We employ classical notations: $h_{K}$ represents the diameter of the triangle $K, h_{e}$ the length of the edge $e$, $h=\max _{K \in \mathcal{T}_{h}} h_{K}$ is the discretization parameter and we denote by $\mathcal{E}_{h}$ the set of all the edges of $\mathcal{T}_{h}$ and by $\mathcal{E}_{h}^{0}$ the set of the internal edges.

We consider the following finite dimensional spaces:

$$
\begin{gathered}
L_{h}=\left\{p^{\prime} \in L^{2}(\Omega) ; p_{\left.\right|_{K}}^{\prime} \in P_{0}, \forall K \in \mathcal{T}_{h}\right\}, \\
V_{h}=\left\{\mathbf{G}^{\prime} \in H(\operatorname{div}, \Omega) ; \mathbf{G}_{\left.\right|_{K}}^{\prime} \in R T_{0}, \forall K \in \mathcal{T}_{h}\right\},
\end{gathered}
$$

where $P_{0}$ is the space of constant functions and $R T_{0}$ is the lowest-order Raviart-Thomas space,

$$
R T_{0}=\left\{\left(\begin{array}{c}
a r+b \\
a z+c
\end{array}\right), a, b, c \in \mathbb{R}\right\}
$$

Then we put

$$
\begin{gathered}
\mathbb{L}_{h}=L_{h} \times L_{h}, \\
\mathbb{V}_{h}^{0}=\left(V_{h} \times V_{h}\right) \cap \mathbb{H}^{0}(\operatorname{div}, \Omega),
\end{gathered}
$$

and we also introduce the affine set:

$$
\mathbb{V}_{h}^{*}=\left\{\left(\mathbf{G}^{\prime}, \mathbf{q}^{\prime}\right) \in V_{h} \times V_{h} \mid \mathbf{G}^{\prime} \cdot \mathbf{n}=\mathcal{I}_{h}(Q) \text { on } \Gamma_{G}, \mathbf{q}^{\prime} \cdot \mathbf{n}=\mathcal{I}_{h}(\Psi) \text { on } \Gamma_{q}\right\}
$$

where $\mathcal{I}_{h}(Q)$ and $\mathcal{I}_{h}(\Psi)$ are piecewise constant approximations of $Q$ on $\Gamma_{G}$, respectively of $\Psi$ on $\Gamma_{q}$.

Let us recall that the solution of the continuous problem satisfies the variational equations:

$$
\left\{\begin{array}{l}
A\left(\mathbf{V}, \mathbf{V}^{\prime}\right)+B\left(s, \mathbf{V}^{\prime}\right)=F_{1}\left(\mathbf{V}^{\prime}\right) \quad \forall \mathbf{V}^{\prime} \in \mathbb{H}^{0}(\operatorname{div}, \Omega) \\
-B\left(s^{\prime}, \mathbf{V}\right)+(C+D)\left(s, s^{\prime}\right)=F_{2}\left(s^{\prime}\right) \quad \forall s^{\prime} \in \mathbb{L}^{2}(\Omega)
\end{array}\right.
$$

where the convective term

$$
D\left(s, s^{\prime}\right)=\int_{\Omega} K(s) T^{\prime} \mathrm{dx}=\int_{\Omega} k \mathbf{G}^{n-1} \cdot \nabla T T^{\prime} \mathrm{dx}+\int_{\Omega} l \mathbf{G}^{n-1} \cdot \nabla p T^{\prime} \mathrm{dx}
$$

is well-defined, thanks to the regularity of the exact solution $\left(\nabla p, \nabla T \in \mathbb{L}^{2}(\Omega)\right)$.

We employ an upwind scheme in order to treat the convective term and we approximate, on every triangle $K \in \mathcal{T}_{h}$ and for every $T \in L_{h}$ :

$$
\int_{K} k \mathbf{G}_{h}^{n-1} \cdot \nabla T \mathrm{dx} \simeq \sum_{e \in \partial K^{-}} k\left(\int_{e} \mathbf{G}_{h}^{n-1} \cdot \mathbf{n} \mathrm{d} \sigma\right)\left(T^{*}-T_{K}\right),
$$

with

$$
\partial K^{-}=\left\{e \in \partial K ; \mathbf{G}_{h}^{n-1} \cdot \mathbf{n}<0\right\}
$$

the set of incoming edges, $T_{K}=T_{\mid K}$ and $T^{*}=T_{\mid K^{*}}$ where the triangle $K^{*}$ is such that $\{e\}=\partial K \cap \partial K^{*}$. 
We recall that since $\mathbf{G}_{h}^{n-1} \in V_{h}$ one has that $\mathbf{G}_{h}^{n-1} \cdot \mathbf{n}$ is constant on every edge.

A similar formula is used for the term $\int_{K} l \mathbf{G}_{h}^{n-1} \cdot \nabla p \mathrm{dx}$.

Whenever the edge $e \in \partial K^{-}$belongs to $\partial \Omega$, we agree to take $T^{*}, p^{*}$ equal to the imposed boundary conditions if $e \subset \Gamma_{T}$ or $e \subset \Gamma_{p}$ and equal to $T_{K}, p_{K}$ otherwise.

We consider the following approximation of $D(\cdot, \cdot)$ on $\mathbb{L}_{h} \times \mathbb{L}_{h}$ :

$$
D_{h}\left(s, s^{\prime}\right)=I_{h}\left(T, T^{\prime}\right)+J_{h}\left(p, T^{\prime}\right)
$$

where the bilinear forms $I_{h}(\cdot, \cdot)$ and $J_{h}(\cdot, \cdot)$ are defined by:

$$
\begin{aligned}
I_{h}\left(T, T^{\prime}\right) & =\sum_{K \in \mathcal{T}_{h}} \sum_{e \in \partial K^{-}} k\left(\int_{e} \mathbf{G}_{h}^{n-1} \cdot \mathbf{n} \mathrm{d} \sigma\right) \delta(T) T_{K}^{\prime}, \\
J_{h}\left(p, T^{\prime}\right) & =\sum_{K \in \mathcal{T}_{h}} \sum_{e \in \partial K^{-}} l\left(\int_{e} \mathbf{G}_{h}^{n-1} \cdot \mathbf{n} \mathrm{d} \sigma\right) \delta(p) T_{K}^{\prime},
\end{aligned}
$$

with

$$
\delta(T)=\left\{\begin{array}{cl}
-T_{K} & \text { if } e \subset \Gamma_{T}, \\
0 & \text { if } e \subset \partial \Omega \backslash \Gamma_{T}, \\
{[T]=T^{*}-T_{K}} & \text { if } e \in \mathcal{E}_{h}^{0}
\end{array}\right.
$$

and with $\delta(p)$ defined in a similar way, with respect to $\Gamma_{p}$. To this bilinear form $D_{h}(\cdot, \cdot)$, we add a linear part corresponding to the non homogeneous boundary conditions:

$$
F_{3 h}\left(s^{\prime}\right)=-\sum_{e \in \partial K^{-} \cap \Gamma_{T}} k\left(\int_{e} \mathbf{G}_{h}^{n-1} \cdot \mathbf{n} \mathrm{d} \sigma\right) T_{\Gamma} T_{K}^{\prime}-\sum_{e \in \partial K^{-} \cap \Gamma_{p}} l\left(\int_{e} \mathbf{G}_{h}^{n-1} \cdot \mathbf{n} \mathrm{d} \sigma\right) p_{\Gamma} T_{K}^{\prime} .
$$

We are now able to write the discrete problem:

$$
\left\{\begin{array}{l}
\text { Find } \mathbf{V}_{h} \in \mathbb{V}_{h}^{*}, s_{h} \in \mathbb{L}_{h} \text { such that } \\
A\left(\mathbf{V}_{h}, \mathbf{V}^{\prime}\right)+B\left(s_{h}, \mathbf{V}^{\prime}\right)=F_{1 h}\left(\mathbf{V}^{\prime}\right) \quad \forall \mathbf{V}^{\prime} \in \mathbb{V}_{h}^{0}, \\
-B\left(s^{\prime}, \mathbf{V}_{h}\right)+\left(C+D_{h}\right)\left(s_{h}, s^{\prime}\right)=F_{2 h}\left(s^{\prime}\right)+F_{3 h}\left(s^{\prime}\right) \quad \forall s^{\prime} \in \mathbb{L}_{h}
\end{array}\right.
$$

where $F_{1 h}(\cdot)$ and $F_{2 h}(\cdot)$ are obtained from $F_{1}(\cdot)$ and $F_{2}(\cdot)$ by replacing $\rho^{n-1}, p^{n-1}, T^{n-1}$ by $\rho_{h}^{n-1}, p_{h}^{n-1}, T_{h}^{n-1}$ respectively.

Remark 3.1. Let us notice that all the coefficients of the discrete problem are obtained, at each time step $t^{n-1}$, from $p^{n-1}$ and $T^{n-1}$ by means of a thermodynamic module. So, in practice, they are piecewise constant functions and this numerical integration introduces a supplementary error of $O(h)$. However, for the sake of simplicity, we neglect here this aspect.

Concerning the continuity of $I_{h}(\cdot, \cdot)$ and $J_{h}(\cdot, \cdot)$, one can prove the following result:

Lemma 3.2. Suppose that $\mathcal{T}_{h}$ satisfies an inverse assumption $h \leq c h_{K}$. Then there exist positive constants $c_{1}$ and $c_{2}$, independent of $h$, such that for any $p, T, T^{\prime} \in L_{h}$ one has:

$$
\begin{gathered}
\left|I_{h}\left(T, T^{\prime}\right)\right| \leq \frac{c_{1}}{h^{2}}\left\|\boldsymbol{G}_{h}^{n-1}\right\|_{0, \Omega}\|T\|_{0, \Omega}\left\|T^{\prime}\right\|_{0, \Omega}, \\
\left|J_{h}\left(p, T^{\prime}\right)\right| \leq \frac{c_{2}}{h^{2}}\left\|\boldsymbol{G}_{h}^{n-1}\right\|_{0, \Omega}\|p\|_{0, \Omega}\left\|T^{\prime}\right\|_{0, \Omega} .
\end{gathered}
$$


Proof. One can write, for any $T, T^{\prime} \in L_{h}$, that:

$$
\begin{aligned}
\left|I_{h}\left(T, T^{\prime}\right)\right| & =\left|\sum_{K \in \mathcal{T}_{h}} \frac{k}{h_{K}}\left(\sum_{e \in \partial K^{-}} \int_{e}\left(\mathbf{G}_{h}^{n-1} \cdot \mathbf{n d} \sigma\right) \delta(T)\right)\left\|T^{\prime}\right\|_{0, K}\right| \\
& \leq \sum_{K \in \mathcal{T}_{h}} \frac{|k|}{h_{K}}\left(\sum_{e \in \partial K^{-}}\left\|\mathbf{G}_{h}^{n-1} \cdot \mathbf{n}\right\|_{0, e}\|\delta(T)\|_{0, e}\right)\left\|T^{\prime}\right\|_{0, K} \\
& \leq \frac{c}{h}\left(\sum_{e \in \mathcal{E}_{h}} h_{e}\left\|\mathbf{G}_{h}^{n-1} \cdot \mathbf{n}\right\|_{0, e}^{2}\right)^{1 / 2}\left(\sum_{e \in \mathcal{E}_{h}} \frac{1}{h_{e}}\|\delta(T)\|_{0, e}^{2}\right)^{1 / 2}\left\|T^{\prime}\right\|_{0, \Omega},
\end{aligned}
$$

by the Cauchy-Schwarz inequality. Using the equivalence of norms in finite dimensional spaces and the passage to the reference finite element, one classically gets for $\mathbf{G}_{h}^{n-1} \in V_{h}$ and $T \in L_{h}$ (see for instance Roberts and Thomas [12], Brenner and Scott [4]) that:

$$
\begin{gathered}
\left(\sum_{e \in \mathcal{E}_{h}} h_{e}\left\|\mathbf{G}^{n-1} \cdot \mathbf{n}\right\|_{0, e}^{2}\right)^{1 / 2} \leq c\left\|\mathbf{G}_{h}^{n-1}\right\|_{0, \Omega}, \\
\left(\sum_{e \in \mathcal{E}_{h}} \frac{1}{h_{e}}\|\delta(T)\|_{0, e}^{2}\right)^{1 / 2} \leq \frac{c}{h}\|T\|_{0, \Omega} .
\end{gathered}
$$

So, the first statement holds. The proof of the second inequality is similar.

\subsection{Existence and uniqueness of a solution}

Let us now study the well-posedness of the discrete problem (14) and apply for that the following variant of the Babuška-Brezzi theorem which can be found in [12].

Besides the inf-sup condition on $B(\cdot, \cdot)$ and the ellipticity of $A(\cdot, \cdot)$ on $\operatorname{Ker}_{h} B$, if the next conditions are satisfied:

(1) $A(\mathbf{V}, \mathbf{V}) \geq 0, \forall \mathbf{V} \in \mathbb{V}_{h}^{0}$

(2) $\left(C+D_{h}\right)(s, s) \geq 0, \forall s \in \mathbb{L}_{h}$,

(3) either $A(\cdot, \cdot)$ or $\left(C+D_{h}\right)(\cdot, \cdot)$ is symmetric,

then the mixed problem (14) has a unique solution.

Moreover, denoting by

$$
\mathcal{A}_{h}=\left[\begin{array}{cc}
A & B \\
-B^{T} & \left(C+D_{h}\right)
\end{array}\right],
$$

it comes that the norm of the inverse matrix $\mathcal{A}_{h}^{-1}$ is independent of the norm of $\left(C+D_{h}\right)$.

In our case, since

$$
\operatorname{Ker}_{h} B=\left\{\left(\mathbf{G}^{\prime}, \mathbf{q}^{\prime}\right) \in \mathbb{V}_{h} ; \operatorname{div} \mathbf{G}^{\prime}=\operatorname{div} \mathbf{q}^{\prime}=0 \text { in } \Omega\right\} \subset \operatorname{Ker} B,
$$

it comes that $A(\cdot, \cdot)$ is $\mathbb{H}(\operatorname{div}, \Omega)$-elliptic on the discrete kernel of $B(\cdot, \cdot)$, with an ellipticity constant independent of $h$. Obviously, $A(\cdot, \cdot)$ is symmetric and positive on the whole space $\mathbb{H}(\operatorname{div}, \Omega)$.

The next lemma ensures that the discrete inf-sup condition on $B(\cdot, \cdot)$ is also satisfied, uniformly with respect to the discretization parameter.

Lemma 3.3. There exists a positive constant $\beta>0$, independent of $h$, such that:

$$
\forall s \in \mathbb{L}_{h}, \sup _{\boldsymbol{V} \in \mathbb{V}_{h}^{0}} \frac{B(s, \boldsymbol{V})}{\mid\|\boldsymbol{V}\|} \geq \beta\|s\|_{0, \Omega} .
$$


Proof. The proof is classical and makes use of the Fortin's trick. That is, with any $s \in \mathbb{L}_{h}$ we associate the couple $\mathbf{V}^{\prime}=\left(\mathbf{G}^{\prime}, \mathbf{q}^{\prime}\right) \in \mathbb{H}^{0}(\operatorname{div}, \Omega)$ solution of the auxiliary problems (9). Since $\Omega$ is a convex polygon, the regularity of the previous elliptic problems gives that $\mathbf{V}^{\prime} \in \mathbb{H}^{1}(\Omega)$, so one may consider its Raviart-Thomas interpolant:

which satisfies:

$$
E_{h}\left(\mathbf{V}^{\prime}\right)=\left(E_{h}\left(\mathbf{G}^{\prime}\right), E_{h}\left(\mathbf{q}^{\prime}\right)\right) \in \mathbb{V}_{h}^{0},
$$

$$
\begin{aligned}
& \left\|\mid E_{h}\left(\mathbf{V}^{\prime}\right)\right\| \leq \leq c\left\|\mathbf{V}^{\prime}\right\|_{1, \Omega} \leq c\|s\|_{0, \Omega} \\
& B\left(s, E_{h}\left(\mathbf{V}^{\prime}\right)\right)=B\left(s, \mathbf{V}^{\prime}\right)=\|s\|_{0, \Omega}^{2}
\end{aligned}
$$

So the lemma is established.

It is now sufficient to prove that $\left(C+D_{h}\right)(\cdot, \cdot)$ is positive on $\mathbb{L}_{h} \times \mathbb{L}_{h}$ which is given in the next lemma.

Lemma 3.4. For $\Delta t$ sufficiently small and for $h \leq c h_{K}$, one has that:

$$
\left(C+D_{h}\right)(s, s) \geq 0, \forall s \in \mathbb{L}_{h}
$$

Proof. Thanks to $(A 3)$, one has that $C(\cdot, \cdot)$ is positive definite:

$$
C(s, s) \geq \frac{c}{\Delta t}\|s\|_{0, \Omega}^{2}, \forall s \in \mathbb{L}^{2}(\Omega) .
$$

Since $\mathbb{L}_{h} \subset \mathbb{L}^{2}(\Omega)$, the previous relation holds on $\mathbb{L}_{h}$, with $c$ independent of $h$ and depending only on the coefficients $a, b, d$ and $f$.

Then, by means of Lemma 3.2, it comes that:

$$
\left(C+D_{h}\right)(s, s) \geq \frac{c}{\Delta t}\left(\|p\|_{0, \Omega}^{2}+\|T\|_{0, \Omega}^{2}\right)-\frac{c_{1}}{h^{2}}\left\|\mathbf{G}_{h}^{n-1}\right\|_{0, \Omega}\|T\|_{0, \Omega}^{2}-\frac{c_{2}}{h^{2}}\left\|\mathbf{G}_{h}^{n-1}\right\|_{0, \Omega}\|p\|_{0, \Omega}\|T\|_{0, \Omega}
$$

So, for

$$
\Delta t \leq \frac{\alpha h^{2}}{\left\|\mathbf{G}_{h}^{n-1}\right\|_{0, \Omega}},
$$

with $\alpha=\frac{2 c}{c_{1}+\sqrt{c_{1}^{2}+c_{2}^{2}}}$, one deduces the announced statement.

Remark 3.5. Let us notice that $I_{h}(\cdot, \cdot)$ is positive (see [2] for the proof), so the above constant $\alpha$ can be taken as $\frac{2 c}{c_{2}}$. Moreover, if one considers $p^{n-1}$ instead of $p^{n}$ in the energy equation, then $D_{h}(\cdot, \cdot)$ becomes positive and there is no condition imposed on $\Delta t$.

So, gathering together the previous results, one deduces

Theorem 3.6. Problem (14) has a unique solution.

Remark 3.7. If we impose a strict inequality in $(15)$, then $\left(C+D_{h}\right)(\cdot, \cdot)$ is even positive definite and the discrete operator $\mathcal{A}_{h}$ is obviously injective. So the discrete problem (14) has a unique solution, whether $B(\cdot, \cdot)$ satisfies the inf-sup condition or not.

Concerning the convergence of the approximation method, let us first notice that one cannot directly apply the results given by the theory of Babuška and Brezzi for mixed formulations, since the continuous problem (5) does not satisfy the Babuška-Brezzi conditions. However, thanks to the well-posedness of the discrete problem, one has:

$$
E^{n}=\left\|\sigma-\sigma_{h}\right\| \leq c_{1} \inf _{\tau_{h} \in \mathbb{V}_{h}^{0} \times \mathbb{L}}\left[\left\|\sigma-\tau_{h}\right\|+\sup _{\tau_{h}^{\prime} \in \mathbb{V}_{h}^{0} \times \mathbb{L}} \frac{\mathcal{D}\left(\sigma, \tau_{h}^{\prime}\right)-\mathcal{D}_{h}\left(\tau_{h}, \tau_{h}^{\prime}\right)+F_{3 h}\left(\tau_{h}^{\prime}\right)}{\left\|\tau_{h}^{\prime}\right\|}\right]+c_{2} E^{n-1},
$$

where $c_{1}, c_{2}$ are independent of time and of the discretization and where the right-hand side term refers to the upwinding scheme. More details can be found in [2]. 


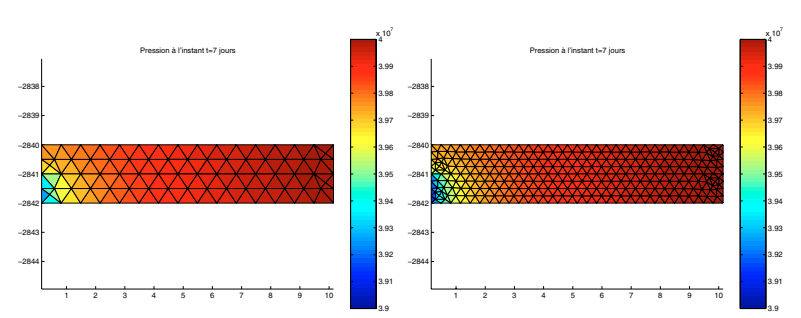

(a) Mesh $\mathcal{T}_{h}$ (b) Mesh $\mathcal{T}_{h / 2}$

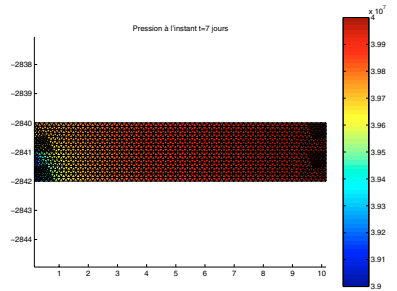

(c) Mesh $\mathcal{T}_{h / 4}$

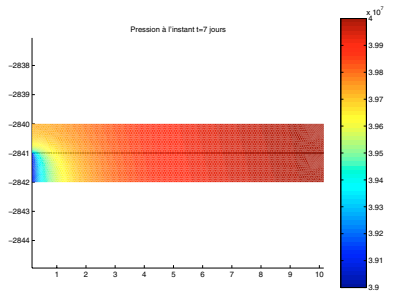

(d) Mesh $\mathcal{T}_{h / 8}$

Figure 3. Pressure maps on congruent meshes at $t=7$ days.

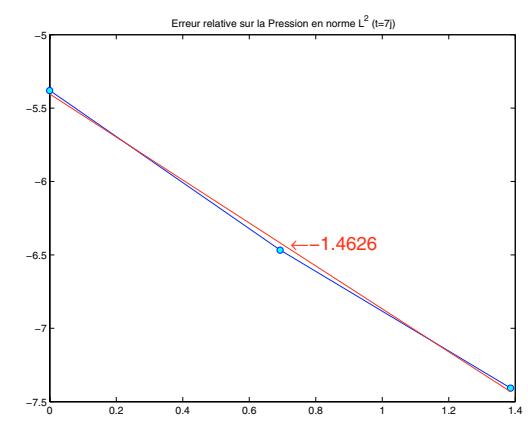

Figure 4. $L^{2}$-Error for the pressure at $t=7$ days.

\section{Numerical Simulations}

In this section we present several numerical tests of our scheme. In order to validate the considered model from both a numerical and a physical point of view, we first study the behaviour of the solution with respect to mesh refinement (Sect. 4.1). We also compare the computed pressure with analytical pressure solutions given by well-test softwares such as PIE (Sect. 4.2). Finally, we present some results obtained for more realistic data (Sect. 4.3).

\subsection{Mesh convergence}

We consider a reservoir divided into two geological layers, which physical and thermodynamic properties are homogeneous (notably $k_{1}=1000 \mathrm{mD}$ and $k_{2}=350 \mathrm{mD}$ ) but where only the lower one is perforated. We intend to simulate the production of a light oil by imposing a difference of pressure $\Delta p=10$ bar between the perforation and the external boundary of the reservoir.

The solutions are computed on congruent meshes $\mathcal{T}_{h / i}$ obtained from an initial mesh $\mathcal{T}_{h}$, each triangle being successively divided into four congruent ones. Note that in order to avoid considerable calculations, the dimensions of the reservoir are deliberately reduced.

We use the solution associated with the finest mesh as a reference solution and we compute the error between this solution and the ones obtained for the intermediate meshes. We have represented the pressure on congruent meshes in Figure 3 and the corresponding error in Figure 4. As shown in Figure 4, we numerically obtain the following error bound for the pressure:

$$
\left\|p-p_{h}\right\|_{0, \Omega} \leq C|h|^{\alpha},
$$

with $\alpha \simeq 1.46$. Similar results hold for the temperature. 


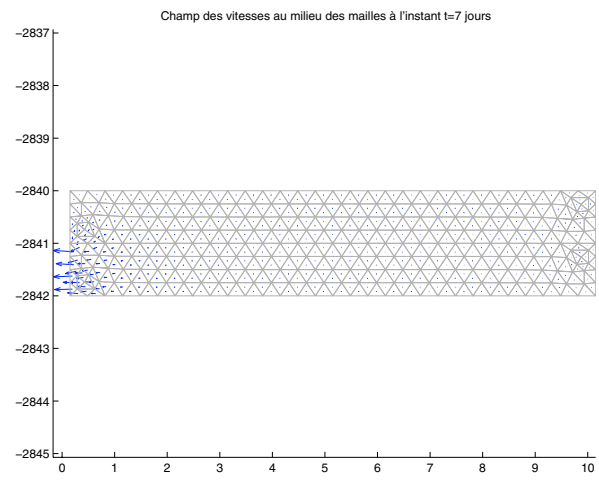

(a) Velocity field

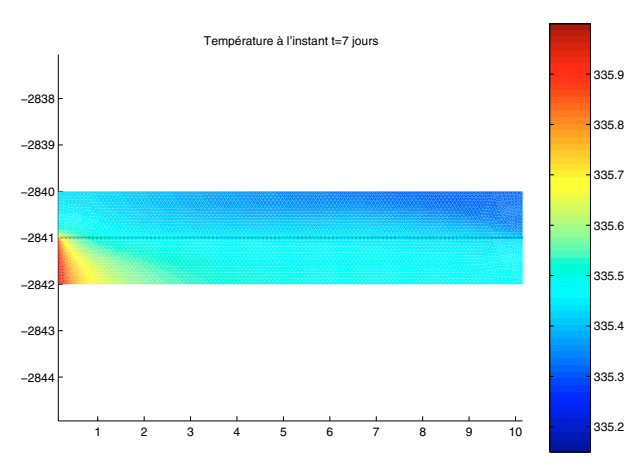

(b) Temperature map

Figure 5. Velocity and temperature on $\mathcal{T}_{h / 2}$ at $t=7$ days.

Figure 5 represents the velocity field and the temperature map after producing during one week.

\subsection{Comparison with well-test softwares}

Well-testing consists in varying the well flow rate, which disturbs the existing pressure in the reservoir, and then in measuring and interpreting the variations in pressure versus time to get information about the reservoir. To make well-test interpretation easier, different softwares such as $\mathrm{PIE}^{1}$ are employed. For a given flow rate history, they are notably able to analytically evaluate the distribution of pressure in the reservoir ( $c f$. [3]) by using Fourier and Laplace transforms. Accordingly, these analytical simulators only work in simplified frameworks and do not take into account the energetic aspect.

Here, our aim is to compare our computed pressure with the one given by PIE.

Thus, we treat the case of a mono-layer reservoir with constant physical and thermodynamic coefficients and with horizontal and vertical permeabilities given by $k_{1}=100 \mathrm{mD}$ and $k_{2}=1 \mathrm{mD}$. Moreover, the flow is supposed to verify the Darcy's assumption and to be independent of the gravity effects, i.e. $F=0$ and $\mathbf{g}=\mathbf{0}$ in (2).

First, we consider a reservoir characterized by a constant pressure (here $p_{\Gamma}=360$ bar) on its external boundary. A production at constant flow rate $\left(Q=150 \mathrm{~m}^{3} /\right.$ day $)$ is simulated during the first 24 hours and is followed by a shut-in period $\left(Q=0 \mathrm{~m}^{3} /\right.$ day $)$ during the next 24 hours. As expected, we observe in Figure $6 \mathrm{a}$ that during the draw-down period, the flow regime goes through a transitory state to reach a permanent one. This permanent state is characterized by a constant wellbore pressure given by:

$$
p_{\text {well }}=p_{\Gamma}-\alpha \frac{Q B \mu}{K h}\left(\ln \frac{R}{r_{w}}+S\right),
$$

where $\alpha$ is a conversion factor, $B$ is the volume factor, $S$ is the skin (null in this experience) and $r_{\mathrm{w}}, R$ respectively refer to the wellbore and to the reservoir radius. Still in permanent regime, we can modify (16) to estimate, at a given $z$, the pressure at any $r$. Figure $6 \mathrm{~b}$ shows that our solution (pink crosses) and the analytical one (in green) are very closed.

Next, we repeat the same experience for a closed reservoir. In other words, a no-flow condition $\mathbf{G} \cdot \mathbf{n}=0$ is now set on the external boundary of the reservoir. As shown in Figure 7 and explained in Bourdarot [3], while the well is producing (the first 24 hours) the flow regime becomes pseudosteady-state as soon as the reservoir boundaries are reached. Moreover, while the well is shut-in (the last 24 hours), the pressure stabilizes at a value called average pressure in the whole area defined by the no-flow boundaries.

\footnotetext{
$1_{\text {WWW.welltestsolutions.com }}$
} 


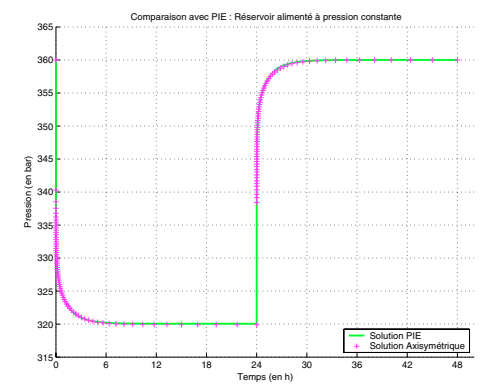

(a) Pressure evolution during $24 \mathrm{~h}$

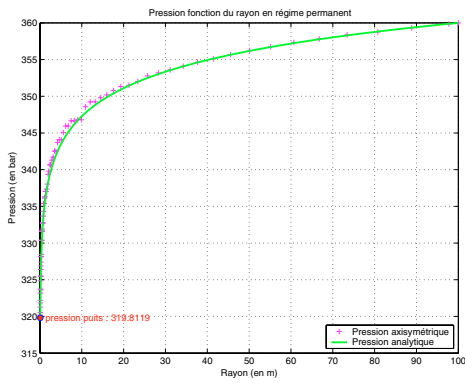

(b) Pressure at a given $z$, in a permanent regime

FiguRE 6. Reservoir with a constant pressure boundary. Comparison of pressures.

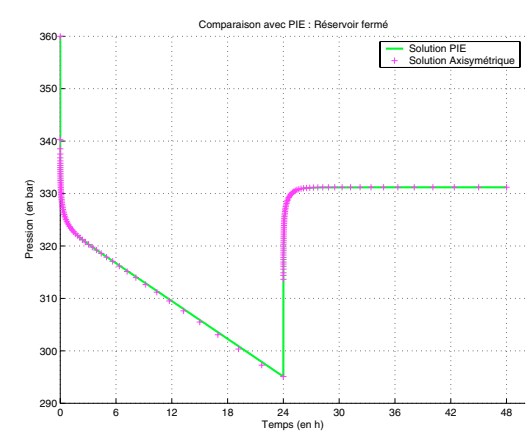

Figure 7. Closed reservoir evolution in time of the pressure near the well.

\subsection{Realistic reservoir}

Let us now present a more realistic example. Our aim is to model an existing reservoir which is divided into seven geological layers characterized by high heterogeneities concerning the physical properties (cf. Fig. 8). Thus, the producing layers (here, the even-numbered ones) have high permeabilities (from 1 to 7 Darcy) and can be separated by quasi-walls (for instance, the third layer from the top) with low porosity and permeability. We simulate the production of a light oil by imposing a gradient of pressure between the perforations and the external boundary of the reservoir.

As one can observe in Figure 9, the temperature and the pressure obtained for different meshes after a one-month production are physically acceptable.

\begin{tabular}{|c|c|c|c|}
\hline Mesh & Nodes & Edges & Triangles \\
\hline Mesh $\mathcal{T}_{1}$ & 173 & 458 & 286 \\
\hline Mesh $\mathcal{T}_{2}$ & 575 & 1609 & 1035 \\
\hline Mesh $\mathcal{T}_{3}$ & 2020 & 5832 & 3813 \\
\hline
\end{tabular}

Figure 10 represents the transitory period at the end of which the pressure is stabilized, while Figure 11 shows the behaviour of the reservoir temperature during a one-month production. 


\begin{tabular}{|llll|}
$k_{1}=7000 \mathrm{mD}$ & $k_{2}=350 \mathrm{mD}$ & $\phi=0.20$ & $s_{w}=0.15$ \\
$k_{1}=7000 \mathrm{mD}$ & $k_{2}=350 \mathrm{mD}$ & $\phi=0.28$ & $s_{w}=0.15$ \\
$k_{1}=10 \mathrm{mD}$ & $k_{2}=1 \mathrm{mD}$ & $\phi=0.08$ & $s_{w}=0.9$ \\
$k_{1}=1000 \mathrm{mD}$ & $k_{2}=15 \mathrm{mD}$ & $\phi=0.24$ & $s_{w}=0.42$ \\
$k_{1}=1000 \mathrm{mD}$ & $k_{2}=15 \mathrm{mD}$ & $\phi=0.26$ & $s_{w}=0.30$ \\
$k_{1}=1000 \mathrm{mD}$ & $k_{2}=15 \mathrm{mD}$ & $\phi=0.22$ & $s_{w}=0.38$ \\
$k_{1}=1000 \mathrm{mD}$ & $k_{2}=15 \mathrm{mD}$ & $\phi=0.24$ & $s_{w}=0.40$ \\
\hline
\end{tabular}

FIGURE 8. Vertical section of the reservoir.

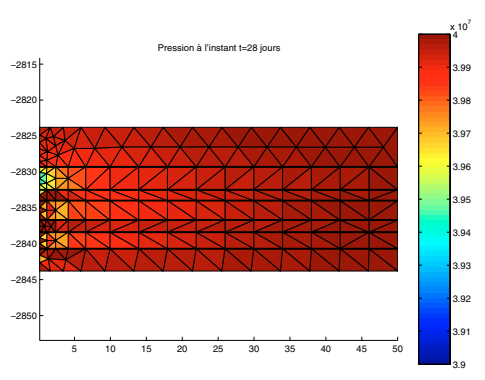

(a) Pressure - Mesh $\mathcal{T}_{1}$

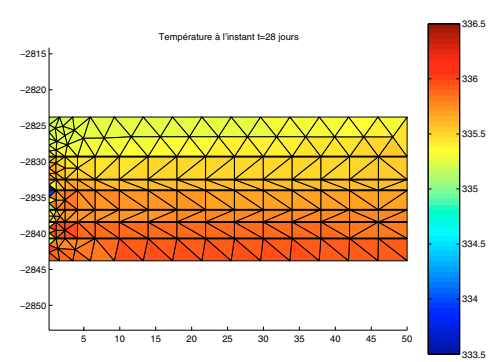

(d) Temperature - Mesh $\mathcal{T}_{1}$

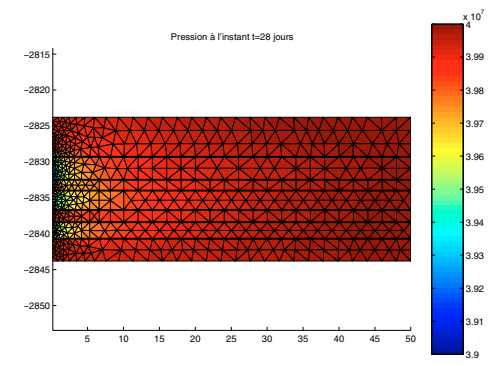

(b) Pressure - Mesh $\mathcal{T}_{2}$

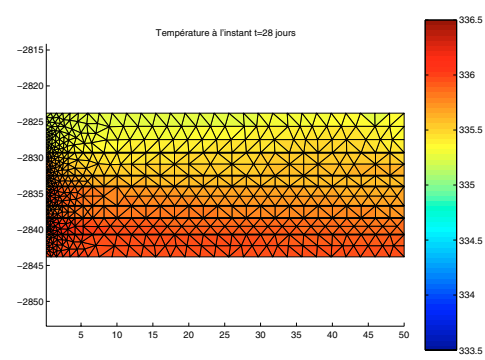

(e) Temperature - Mesh $\mathcal{T}_{2}$

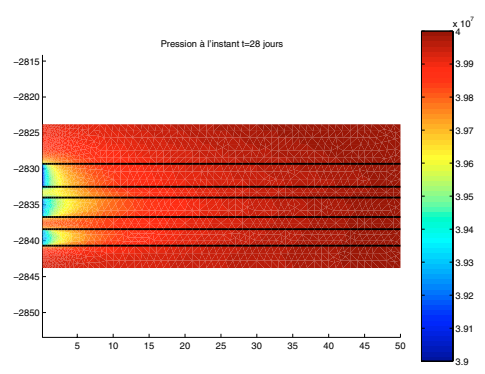

(c) Pressure - Mesh $\mathcal{T}_{3}$

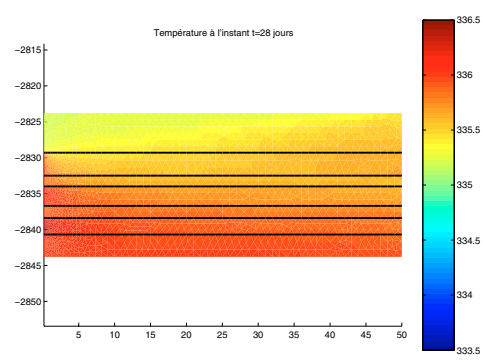

(f) Temperature - Mesh $\mathcal{T}_{3}$

Figure 9. Pressure and temperature maps at $t=28$ days, for realistic data.

\subsection{Influence of the permeability}

As one can notice from the previous examples, when treating a reservoir associated with a vertical wellbore, one generally has that $k_{1} \gg k_{2}$ where $k_{1}$ is the radial permeability and $k_{2}$ the vertical one. However, in order to numerically validate the code, we next present an example where $k_{2} \gg k_{1}$. More precisely, we take $k_{1}=1 \mathrm{mD}$ whereas $k_{2}=1000 \mathrm{mD}$. Since the flow is mainly radial, the influence of the radial permeability $k_{1}$ is preponderant compared with the one of the vertical permeability $k_{2}$, which leads to similar results from a qualitative point of view for both situations $k_{2} \gg k_{1}$ and $k_{2} \ll k_{1}$ (cf. Fig. 12). 


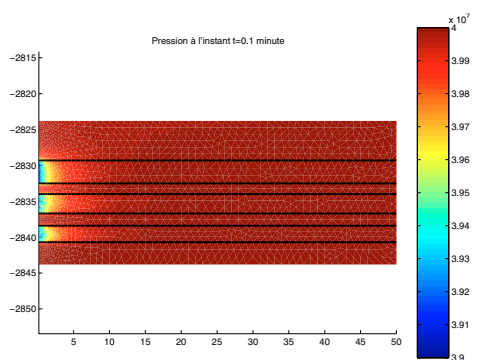

(a)

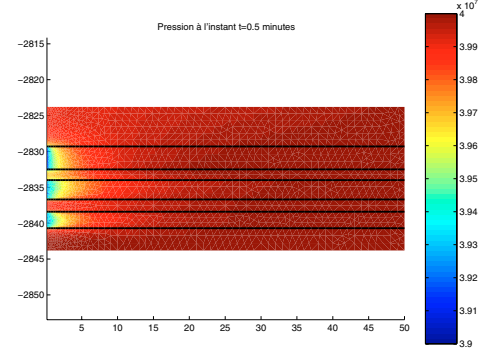

(b)

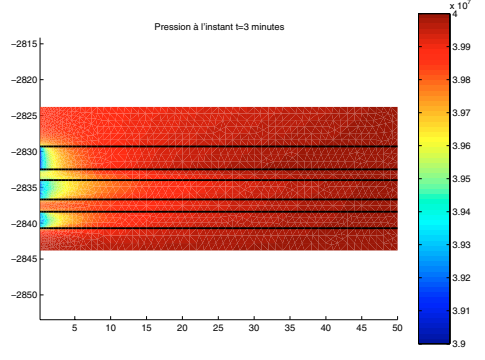

(c)

FiguRE 10. The pressure reaching steady state.

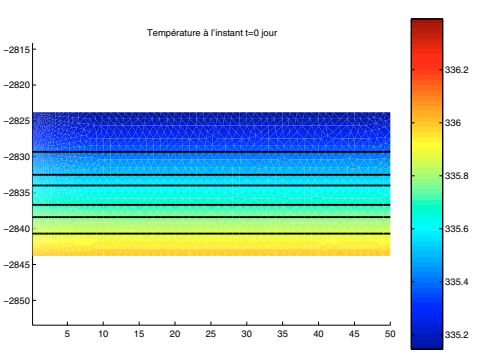

(a) $\mathrm{t}=0$ day

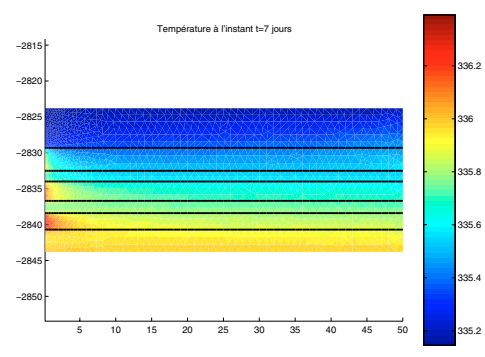

(b) $t=7$ days

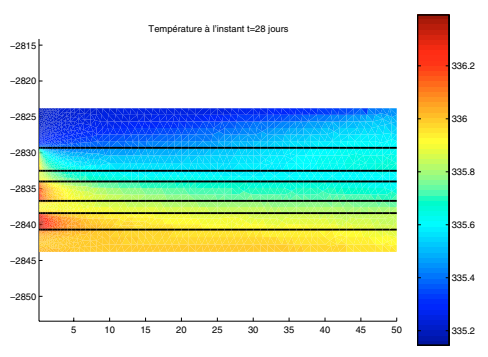

(c) $\mathrm{t}=28$ days

FiguRE 11. Behaviour of the reservoir temperature during a one-month production.

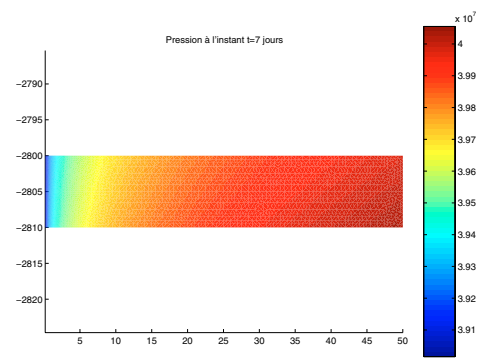

(a) Pressure

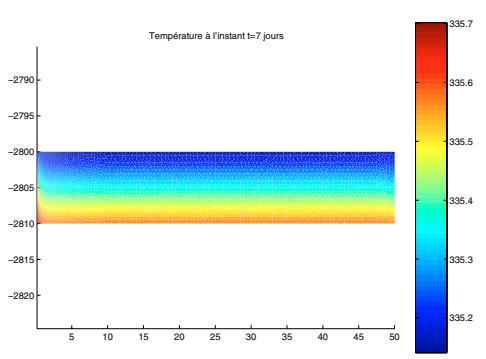

(b) Temperature

FiguRE 12. A one-layer reservoir with $k_{2} \gg k_{1}$.

Finally, we detail an example where the permeability is extremely different in the layers. This aspect was already encountered to a lesser extent when studying the realistic case.

We take for that a two-layer reservoir with $\left(k_{1}, k_{2}\right)=(5000 \mathrm{mD}, 350 \mathrm{mD})$ for the first layer and $(1 \mathrm{mD}, 1 \mathrm{mD})$ for the second one, and we impose a difference of pressure between the perforation and the external boundary of the reservoir. The layers are both perforated but due to a better permeability, the flow mainly takes place through the first layer, which leads to a higher rise in the temperature as shown in Figure 13. 


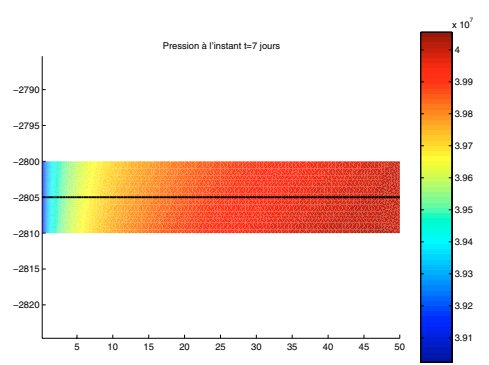

(a) Pressure

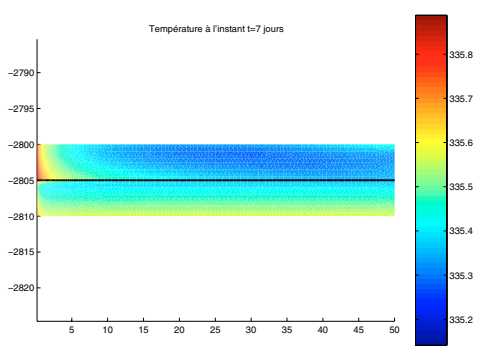

(b) Temperature

FiguRE 13. Two-layer reservoir with heterogeneous permeability.

\section{A POSTERIORI ERROR ESTIMATORS}

We define in this section reliable and efficient a posteriori estimators, which can be used for local mesh refinements and implicitly for improvement of the solution.

It is useful to write the discrete problem (14) under the equivalent form:

$$
\left\{\begin{array}{l}
\text { Find } \sigma_{h} \in \mathbb{V}_{h}^{*} \times \mathbb{L}_{h} \\
\mathcal{A}_{h}\left(\sigma_{h}, \sigma_{h}^{\prime}\right)=\mathcal{F}_{h}\left(\sigma_{h}^{\prime}\right), \quad \forall \sigma_{h}^{\prime} \in \mathbb{V}_{h}^{0} \times \mathbb{L}_{h},
\end{array}\right.
$$

where $\sigma_{h}=\left(\mathbf{V}_{h}, s_{h}\right), \mathcal{A}_{h}=\left[\begin{array}{cc}A & B \\ -B^{T} & \left(C+D_{h}\right)\end{array}\right]$ and $\mathcal{F}_{h}=\left(\begin{array}{c}F_{1 h} \\ F_{2 h}+F_{3 h}\end{array}\right)$.

\subsection{Mesh-dependent norms and error indicators}

In order to define a posteriori error indicators on the Raviart-Thomas finite element space $\mathbb{V}_{h}$, we apply some results of Verfürth and Braess [13]. We begin by introducing the following mesh-dependent norms:

$$
\begin{aligned}
& \|\mid \mathbf{V}\|_{h}=\left(\|\mathbf{G}\|_{0, \Omega}^{2}+\|\mathbf{q}\|_{0, \Omega}^{2}+\sum_{e \in \mathcal{E}_{h}} h_{e}\|\mathbf{G} \cdot \mathbf{n}\|_{0, e}^{2}+\sum_{e \in \mathcal{E}_{h}} h_{e}\|\mathbf{q} \cdot \mathbf{n}\|_{0, e}^{2}\right)^{1 / 2}, \forall \mathbf{V}=(\mathbf{G}, \mathbf{q}) \in \mathbb{V}_{h}^{0}, \\
& |s|_{1, h}=\left(\sum_{K \in \mathcal{T}_{h}}|p|_{1, K}^{2}+\sum_{K \in \mathcal{T}_{h}}|T|_{1, K}^{2}+\sum_{e \in \mathcal{E}_{h}} h_{e}^{-1}\|[p]\|_{0, e}^{2}+\sum_{e \in \mathcal{E}_{h}} h_{e}^{-1}\|[T]\|_{0, e}^{2}\right)^{1 / 2}, \forall s=(p, T) \in \mathbb{L}_{h},
\end{aligned}
$$

where $[p]$ represents the jump of $p$ across an internal edge $e$, respectively the trace of $p$ if $e \subset \partial \Omega$.

Then the following equivalences of norms hold:

$$
\begin{gathered}
\|\mathbf{V}\|_{0, \Omega} \leq\||| \mathbf{V}\|_{h} \leq c\|\mathbf{V}\|_{0, \Omega}, \quad \forall \mathbf{V} \in \mathbb{V}_{h}^{0}, \\
\|s\|_{0, \Omega} \leq c h|s|_{1, h} \leq c^{\prime}\|s\|_{0, \Omega}, \quad \forall s \in \mathbb{L}_{h},
\end{gathered}
$$

with $c, c^{\prime}$ independent of $h$. We refer for instance to Roberts and Thomas [12] for the proof of (19) and (20). 
Moreover, one has:

$$
\begin{gathered}
|B(s, \mathbf{V})| \leq c|||\mathbf{V}|||_{h}|s|_{1, h}, \forall \mathbf{V} \in \mathbb{V}_{h}^{0}, \forall s \in \mathbb{L}_{h}, \\
\sup _{\mathbf{V} \in \mathbb{V}_{h}^{0}} \frac{B(s, \mathbf{V})}{\||\mathbf{V}|\|_{h}} \geq c|s|_{1, h}, \forall s \in \mathbb{L}_{h},
\end{gathered}
$$

where the first statement is obvious and the second one is established, for instance, in Verfürth and Braess [13].

Finally, the above inequalities ensure that the bilinear forms $A(\cdot, \cdot)$ and $B(\cdot, \cdot)$ satisfy the Babuška-Brezzi conditions with respect to the discrete norms $\left.|||\cdot|\right|_{h}$ and $|\cdot|_{1, h}$.

Concerning the continuity of $\left(C+D_{h}\right)(\cdot, \cdot)$ with respect to the norm $|\cdot|_{1, h}$, one can easily show that:

$$
C\left(s, s^{\prime}\right)+D_{h}\left(s, s^{\prime}\right) \leq\left(\frac{c_{1} h^{2}}{\Delta t}+c_{2}\left\|\mathbf{G}_{h}^{n-1}\right\|_{0, \Omega}\right)|s|_{1, h}\left|s^{\prime}\right|_{1, h}, \forall s, s^{\prime} \in \mathbb{L}_{h},
$$

therefore its continuity constant depends on $\frac{h^{2}}{\Delta t}$. However, the norm of the inverse operator $\mathcal{A}_{h}^{-1}$ being independent of the norm of $C+D_{h}$, the following stability property is true:

$$
\||\mathbf{V}|\|_{h}+|s|_{1, h} \leq c \sup _{\left(\mathbf{V}^{\prime}, s^{\prime}\right) \in \mathbb{V}_{h}^{0} \times \mathbb{L}_{h}} \frac{\mathcal{A}_{h}\left((\mathbf{V}, s),\left(\mathbf{V}^{\prime}, s^{\prime}\right)\right)}{\left\|\left|\mathbf{V}^{\prime} \|\right|_{h}+\left|s^{\prime}\right|_{1, h}\right.}, \forall(\mathbf{V}, s) \in \mathbb{V}_{h}^{0} \times \mathbb{L}_{h}
$$

with a constant $c$ which only depends on the thermodynamic coefficients.

Let us proceed with the a posteriori error analysis. For that, we introduce the following residuals on any triangle $K \in \mathcal{T}_{h}$ and any edge $e \in \mathcal{E}_{h}$ :

$$
\begin{gathered}
\eta_{K, 1}=\left(\left\|\frac{1}{r} \underline{\mathbf{M}} \mathbf{G}_{h}+\nabla p_{h}-\rho_{h}^{n-1} \mathbf{g}\right\|_{0, K}^{2}+\left\|\frac{1}{r \lambda} \mathbf{q}_{h}-\nabla T_{h}\right\|_{0, K}^{2}\right)^{1 / 2} \\
\eta_{K, 2}=\left(\left\|r \frac{a}{\Delta t} p_{h}-r \frac{b}{\Delta t} T_{h}+\operatorname{div} \mathbf{G}_{h}-r \frac{a}{\Delta t} p_{h}^{n-1}+r \frac{b}{\Delta t} T_{h}^{n-1}\right\|_{0, K}^{2}\right. \\
+\| r \frac{d}{\Delta t} T_{h}-r \frac{f}{\Delta t} p_{h}+\frac{k}{|K|} \sum_{e \in \partial K^{-}} \int_{e}\left(\mathbf{G}_{h}^{n-1} \cdot \mathbf{n}\right) \eta_{e, 2} \mathrm{~d} \sigma \\
\left.+\frac{l}{|K|} \sum_{e \in \partial K^{-}} \int_{e}\left(\mathbf{G}_{h}^{n-1} \cdot \mathbf{n}\right) \eta_{e, 1} \mathrm{~d} \sigma-\operatorname{div}_{h}-r \frac{d}{\Delta t} T_{h}^{n-1}+r \frac{f}{\Delta t} p_{h}^{n-1} \|_{0, K}^{2}\right)^{1 / 2}, \\
\eta_{e}=\left(\left\|\eta_{e, 1}\right\|_{0, e}^{2}+\left\|\eta_{e, 2}\right\|_{0, e}^{2}\right)^{1 / 2}
\end{gathered}
$$

where

$$
\eta_{e, 1}=\left\{\begin{array}{cl}
p_{h}-p_{\Gamma} & \text { if } e \subset \Gamma_{p} \\
0 & \text { if } e \subset \partial \Omega \backslash \Gamma_{p} \\
{\left[p_{h}\right]} & \text { if } e \in \mathcal{E}_{h}^{0}
\end{array}, \eta_{e, 2}=\left\{\begin{array}{cl}
T_{h}-T_{\Gamma} & \text { if } e \subset \Gamma_{T} \\
0 & \text { if } e \subset \partial \Omega \backslash \Gamma_{T} \\
{\left[T_{h}\right]} & \text { if } e \in \mathcal{E}_{h}^{0}
\end{array}\right.\right.
$$


The local error indicator is a weighted combination:

$$
\eta_{K}=\left(\eta_{K, 1}^{2}+h_{K}^{2} \eta_{K, 2}^{2}+\sum_{e \in \partial K} h_{e}^{-1} \eta_{e}^{2}\right)^{1 / 2}
$$

and the global one is given by:

$$
\eta=\left[\sum_{K \in \tau_{h}}\left(\eta_{K, 1}^{2}+h_{K}^{2} \eta_{K, 2}^{2}\right)+\sum_{e \in \mathcal{E}_{h}} h_{e}^{-1} \eta_{e}^{2}\right]^{1 / 2}
$$

According to [13], we make the following saturation assumption $(S A): \exists \beta<1$ such that

$$
|| \mathbf{V}-\left.\mathbf{V}_{h / 2}||\right|_{h / 2}+\left|s-s_{h / 2}\right|_{1, h / 2} \leq \beta\left(\left.||\left|\mathbf{V}-\mathbf{V}_{h}\right|\right|_{h / 2}+\left|s-s_{h}\right|_{1, h / 2}\right)
$$

where $\mathcal{T}_{h / 2}$ is obtained from $\mathcal{T}_{h}$ by dividing each triangle into four congruent ones and where $\sigma_{h / 2}=\left(\mathbf{V}_{h / 2}, s_{h / 2}\right)$ is the unique solution of the variational problem:

$$
\left\{\begin{array}{l}
\text { Find } \sigma_{h / 2} \in \mathbb{V}_{h / 2}^{*} \times \mathbb{L}_{h / 2} \\
\mathcal{A}_{h / 2}\left(\sigma_{h / 2}, \sigma^{\prime}\right)=\mathcal{F}_{h / 2}\left(\sigma^{\prime}\right), \forall \sigma^{\prime} \in \mathbb{V}_{h / 2}^{0} \times \mathbb{L}_{h / 2}
\end{array}\right.
$$

with $\mathcal{A}_{h / 2}=\left[\begin{array}{cc}A & B \\ -B^{T} & C+D_{h / 2}\end{array}\right]$ and $\mathcal{F}_{h / 2}=\left(\begin{array}{c}F_{1 h} \\ F_{2 h}+F_{3 h / 2}\end{array}\right)$.

We took the same right-hand side terms $F_{1 h}, F_{2 h}$ as in (17), since they only depend on discrete quantities already computed at $t^{n-1}$, but we redefined on $\mathcal{T}_{h / 2}$ the terms arising from the upwind scheme.

\subsection{Error analysis}

We first establish an upper bound for the error. One immediately gets (see for instance [13]):

$$
\begin{gathered}
\left\|\left.\left|\mathbf{G}^{\prime}\right|\right|_{h / 2} \leq\right\|\left|\mathbf{G}^{\prime}\right| \|_{h} \leq\left.\sqrt{2}||\left|\mathbf{G}^{\prime}\right|\right|_{h / 2}, \quad \forall \mathbf{G}^{\prime} \in V_{h}, \\
\frac{1}{\sqrt{2}}\left|p^{\prime}\right|_{1, h / 2} \leq\left|p^{\prime}\right|_{1, h} \leq\left|p^{\prime}\right|_{1, h / 2}, \quad \forall p^{\prime} \in L_{h} .
\end{gathered}
$$

Then the following statement holds:

Theorem 5.1. One has that:

$$
\|\left.\left|\boldsymbol{V}_{h / 2}-\boldsymbol{V}_{h}\right|\right|_{h}+\left|s_{h / 2}-s_{h}\right|_{1, h / 2} \leq c \eta,
$$

with $c$ independent of the discretization and of $\Delta t$.

Proof. Using the stability property (23) for the problem (24) and the fact that $\mathbf{V}_{h}-\mathbf{V}_{h / 2} \in \mathbb{V}_{h}^{0}$, one has that:

$$
\|\left|\mathbf{V}_{h}-\mathbf{V}_{h / 2}\right|||_{h / 2}+\left|s_{h}-s_{h / 2}\right|_{1, h / 2} \leq c \sup _{\left(\mathbf{V}^{\prime}, s^{\prime}\right) \in \mathbb{V}_{h / 2}^{0} \times \mathbb{L}_{h / 2}} \frac{\mathcal{A}_{h / 2}\left(\left(\mathbf{V}_{h}-\mathbf{V}_{h / 2}, s_{h}-s_{h / 2}\right),\left(\mathbf{V}^{\prime}, s^{\prime}\right)\right)}{\left\|\left.\left|\mathbf{V}^{\prime} \|_{h / 2}+\right| s^{\prime}\right|_{1, h / 2}\right.}
$$


Let us estimate:

$$
\begin{aligned}
\mathcal{A}_{h / 2}\left(\left(\mathbf{V}_{h}-\mathbf{V}_{h / 2}, s_{h}-s_{h / 2}\right),\left(\mathbf{V}^{\prime}, s^{\prime}\right)\right)=A\left(\mathbf{V}_{h}, \mathbf{V}^{\prime}\right)+ & B\left(s_{h}, \mathbf{V}^{\prime}\right)-B\left(s^{\prime}, \mathbf{V}_{h}\right) \\
& +\left(C+D_{h / 2}\right)\left(s_{h}, s_{h / 2}^{\prime}\right)-F_{1 h}\left(\mathbf{V}^{\prime}\right)-F_{2 h}\left(s^{\prime}\right)-F_{3 h / 2}\left(s^{\prime}\right) .
\end{aligned}
$$

For that, we integrate by parts:

$$
B\left(s_{h}, \mathbf{V}^{\prime}\right)=\sum_{e \in \mathcal{E}_{h}^{0} \cup \Gamma_{p}} \int_{e} \mathbf{G}^{\prime} \cdot \mathbf{n}\left[p_{h}\right] \mathrm{d} \sigma-\sum_{e \in \mathcal{E}_{h}^{0} \cup \Gamma_{T}} \int_{e} \mathbf{q}^{\prime} \cdot \mathbf{n}\left[T_{h}\right] \mathrm{d} \sigma
$$

and we take into account the boundary terms of $F_{1 h}(\cdot)$. The Cauchy-Schwarz inequality then gives that:

$$
\begin{aligned}
A\left(\mathbf{V}_{h}, \mathbf{V}^{\prime}\right)+B & \left(s_{h}, \mathbf{V}^{\prime}\right)-F_{1 h}\left(\mathbf{V}^{\prime}\right) \\
& \leq \sum_{K \in \mathcal{T}_{h}} \eta_{1, K}\left\|\mathbf{V}^{\prime}\right\|_{0, K}+\left(\sum_{e \in \mathcal{E}_{h}} h_{e}\left\|\mathbf{G}^{\prime} \cdot \mathbf{n}\right\|_{0, e}^{2}+h_{e}\left\|\mathbf{q}^{\prime} \cdot \mathbf{n}\right\|_{0, e}^{2}\right)^{1 / 2}\left(\sum_{e \in \mathcal{E}_{h}} h_{e}^{-1} \eta_{e}^{2}\right)^{1 / 2} \\
& \leq\left(\sum_{K \in \mathcal{T}_{h}} \eta_{1, K}^{2}+\sum_{e \in \mathcal{E}_{h}} h_{e}^{-1} \eta_{e}^{2}\right)^{1 / 2}\left(\left\|\mathbf{V}^{\prime}\right\|_{0, \Omega}^{2}+\sum_{e \in \mathcal{E}_{h}} h_{e}\left\|\mathbf{G}^{\prime} \cdot \mathbf{n}\right\|_{0, \Omega}^{2}+h_{e}\left\|\mathbf{q}^{\prime} \cdot \mathbf{n}\right\|_{0, \Omega}^{2}\right)^{1 / 2} \leq \eta\left\|\mathbf{V}^{\prime}\right\| \|_{h} .
\end{aligned}
$$

In the same manner, one may write that:

$$
\begin{aligned}
-B\left(s^{\prime}, \mathbf{V}_{h}\right) & +\left(C+D_{h / 2}\right)\left(s_{h}, s^{\prime}\right)-F_{2 h}\left(s^{\prime}\right)-F_{3 h / 2}\left(s^{\prime}\right) \\
& =-B\left(s^{\prime}, \mathbf{V}_{h}\right)+\left(C+D_{h}\right)\left(s_{h}, s^{\prime}\right)-F_{2 h}\left(s^{\prime}\right)-F_{3 h}\left(s^{\prime}\right)+\left(D_{h / 2}-D_{h}\right)\left(s_{h}, s^{\prime}\right)+\left(F_{3 h}-F_{3 h / 2}\right)\left(s^{\prime}\right) \\
& \leq \sum_{K \in \mathcal{T}_{h}} \eta_{2, K}\left\|s^{\prime}\right\|_{0, \Omega}+\left(D_{h / 2}-D_{h}\right)\left(s_{h}, s^{\prime}\right)+\left(F_{3 h}-F_{3 h / 2}\right)\left(s^{\prime}\right) \\
& \leq\left(\sum_{K \in \mathcal{T}_{h}} h_{K}^{2} \eta_{2, K}^{2}\right)^{1 / 2}\left|s^{\prime}\right|_{1, h}+\left(D_{h / 2}-D_{h}\right)\left(s_{h}, s^{\prime}\right)+\left(F_{3 h}-F_{3 h / 2}\right)\left(s^{\prime}\right) .
\end{aligned}
$$

By using the continuity of $D_{h}(\cdot, \cdot)$ and $F_{3 h}(\cdot)$ (see the proof of Lem. 3.2) and the relations (25) and (26), one has that:

$$
D_{h}\left(s_{h}, s^{\prime}\right)-F_{3 h}\left(s^{\prime}\right) \leq c\left\|\mathbf{G}_{h}^{n-1}\right\|_{0, \Omega}\left|s^{\prime}\right|_{1, h / 2}\left(\sum_{e \in \mathcal{E}_{h}} h_{e}^{-1} \eta_{e}^{2}\right)^{1 / 2}
$$

and similarly,

$$
D_{h / 2}\left(s_{h}, s^{\prime}\right)-F_{3 h / 2}\left(s^{\prime}\right) \leq c^{\prime}\left\|\mathbf{G}_{h}^{n-1}\right\|_{0, \Omega}\left|s^{\prime}\right|_{1, h / 2}\left(\sum_{e \in \mathcal{E}_{h}} h_{e}^{-1} \eta_{e}^{2}\right)^{1 / 2}
$$

Therefore, it now comes that:

$$
-B\left(s^{\prime}, V_{h}\right)+\left(C+D_{h / 2}\right)\left(s_{h}, s^{\prime}\right)-F_{2 h}\left(s^{\prime}\right)-F_{3 h / 2}\left(s^{\prime}\right) \leq c\left(\sum_{K \in \mathcal{T}_{h}} h_{K}^{2} \eta_{2, K}^{2}+\sum_{e \in \mathcal{E}_{h}} h_{e}^{-1} \eta_{e}^{2}\right)^{1 / 2}\left|s^{\prime}\right|_{1, h / 2},
$$


and finally:

$$
\mathcal{A}_{h / 2}\left(\left(\mathbf{V}_{h}-\mathbf{V}_{h / 2}, s_{h}-s_{h / 2}\right),\left(\mathbf{V}^{\prime}, s^{\prime}\right)\right) \leq c \eta\left(\left|s^{\prime}\right|_{1, h / 2}+\left\|\mid \mathbf{V}^{\prime}\right\|_{h / 2}\right)
$$

with $c$ depending on $\left\|\mathbf{G}_{h}^{n-1}\right\|_{0, \Omega}$. This last estimate leads to the conclusion.

By applying the triangle inequality and the saturation assumption $(S A)$, one can now deduce an upper estimate for the error in weighted norms:

Theorem 5.2. The following error bound holds:

$$
\|\left.\left|\boldsymbol{V}-\boldsymbol{V}_{h}\right|\right|_{h}+\left|s-s_{h}\right|_{1, h} \leq \frac{c}{1-\beta} \eta
$$

Concerning a lower bound for the error, this is easily obtained thanks to the definition of the discrete weighted norms. Indeed, the regularity of the continuous time-discretized problem (7) gives $s \in \mathbb{H}^{1}(\Omega)$, therefore by the trace theorem one gets that $\left[s_{h}\right]=\left[s_{h}-s\right]$ on every internal edge $e \in \mathcal{E}_{h}^{0}$. So,

$$
\left(\sum_{e \in \mathcal{E}_{h}} h_{e}^{-1} \eta_{e}^{2}\right)^{1 / 2} \leq\left|s_{h}-s\right|_{1, h}
$$

Moreover, since the exact solution $(\mathbf{V}, s)$ satisfies:

$$
\frac{1}{r} \underline{\mathbf{M G}}+\nabla p-\rho^{n-1} \mathbf{g}=0 \text { and } \frac{1}{r \lambda} \mathbf{q}-\nabla T=0 \text { a.e. in } \Omega
$$

one may write that:

$$
\begin{aligned}
\eta_{K, 1}^{2} & =\left\|\nabla\left(p-p_{h}\right)+\frac{1}{r} \underline{\mathbf{M}}\left(\mathbf{G}-\mathbf{G}_{h}\right)-\left(\rho^{n-1}-\rho_{h}^{n-1}\right) \mathbf{g}\right\|_{0, K}^{2}+\left\|\nabla\left(T-T_{h}\right)+\frac{1}{r \lambda}\left(\mathbf{q}-\mathbf{q}_{h}\right)\right\|_{0, K}^{2} \\
& \leq c\left(\left|p-p_{h}\right|_{1, K}^{2}+\left|T-T_{h}\right|_{1, K}^{2}+\left\|\mathbf{G}-\mathbf{G}_{h}\right\|_{0, K}^{2}+\left\|\mathbf{q}-\mathbf{q}_{h}\right\|_{0, K}^{2}+\left\|\rho^{n-1}-\rho_{h}^{n-1}\right\|_{0, K}^{2}\right) .
\end{aligned}
$$

Hence,

$$
\left(\sum_{K \in \mathcal{T}_{h}} \eta_{K, 1}^{2}\right)^{1 / 2} \leq c\left(\left|s-s_{h}\right|_{1, h}+\left\|\left|\mathbf{V}-\mathbf{V}_{h}\right|_{\|_{h}}+\right\| \rho^{n-1}-\rho_{h}^{n-1} \|_{0, \Omega}\right) .
$$

In order to bound the last residual $\eta_{K, 2}$, we use that $\operatorname{div} \mathbf{G}_{h} \in L_{h}$, for all $\mathbf{G}_{h} \in V_{h}$. Then the last variational equation of (14) gives, on every $K \in \mathcal{T}_{h}$ :

$$
\begin{aligned}
& \operatorname{div} \mathbf{G}_{h}=\mathcal{P}_{h}\left(\frac{r a}{\Delta t} p_{h}^{n-1}-\frac{r b}{\Delta t} T_{h}^{n-1}\right)-\mathcal{P}_{h}\left(\frac{r a}{\Delta t} p_{h}-\frac{r b}{\Delta t} T_{h}\right) \\
\operatorname{divq}_{h}= & \mathcal{P}_{h}\left(\frac{r d}{\Delta t} T_{h}-\frac{r f}{\Delta t} p_{h}\right)-\mathcal{P}_{h}\left(\frac{r d}{\Delta t} T_{h}^{n-1}-\frac{r f}{\Delta t} p_{h}^{n-1}\right) \\
& +\frac{k}{|K|} \sum_{e \in K^{-}} \int_{e}\left(\mathbf{G}_{h}^{n-1} \cdot \mathbf{n}\right) \eta_{e, 2} \mathrm{~d} \sigma+\frac{l}{|K|} \sum_{e \in \partial K^{-}} \int_{e}\left(\mathbf{G}_{h}^{n-1} \cdot \mathbf{n}\right) \eta_{e, 1} \mathrm{~d} \sigma,
\end{aligned}
$$

with $\mathcal{P}_{h}: L^{2}(\Omega) \longrightarrow L_{h}$ the piecewise constant $L^{2}$-orthogonal projection operator. 
Then by replacing $\operatorname{divq}_{h}$ and $\operatorname{div} \mathbf{G}_{h}$ in $\eta_{K, 2}$, it comes that:

$$
\begin{aligned}
\eta_{K, 2}^{2} \leq & \left\|\left(\frac{a}{\Delta t} p_{h}^{n-1}-\frac{b}{\Delta t} T_{h}^{n-1}-\frac{a}{\Delta t} p_{h}+\frac{b}{\Delta t} T_{h}\right)\left(r-\mathcal{P}_{h} r\right)\right\|_{0, K}^{2} \\
& +\left\|\left(\frac{d}{\Delta t} T_{h}-\frac{f}{\Delta t} p_{h}-\frac{d}{\Delta t} T_{h}^{n-1}+\frac{f}{\Delta t} p_{h}^{n-1}\right)\left(r-\mathcal{P}_{h} r\right)\right\|_{0, K}^{2} \\
\leq & \frac{c}{\Delta t^{2}}\left\|r-\mathcal{P}_{h} r\right\|_{0, K}^{2}
\end{aligned}
$$

and finally,

$$
\left(\sum_{K \in \mathcal{T}_{h}} h^{2} \eta_{K, 2}^{2}\right)^{1 / 2} \leq \frac{c h^{2}}{\Delta t}
$$

By putting together (27), (28) and (29), we have thus established:

Theorem 5.3. The error estimator $\eta$ yields the following global, respectively local, lower bounds:

$$
\begin{gathered}
\eta \leq c\left(||\left|\boldsymbol{V}-\boldsymbol{V}_{h}\right|\left\|_{h}+\left|s-s_{h}\right|_{1, h}+\right\| \rho_{h}^{n-1}-\rho^{n-1} \|_{0, \Omega}\right)+\frac{c h^{2}}{\Delta t} \\
\eta_{K} \leq c\left(||\left|\boldsymbol{V}-\boldsymbol{V}_{h}\right|\left\|_{h, \omega_{K}}+\left|s-s_{h}\right|_{1, h, \omega_{K}}+\right\| \rho_{h}^{n-1}-\rho^{n-1} \|_{0, K}\right)+\frac{c h^{2}}{\Delta t}
\end{gathered}
$$

where $\omega_{K}$ is the set of the triangles $K^{\prime} \in \mathcal{T}_{h}$, neighbours of $K$ and $c$ is independent of $h$ and $\Delta t$.

Remark 5.4. One can also consider a posteriori error estimates with respect to the natural norms of $\mathbb{H}(\operatorname{div}, \Omega)$ and $\mathbb{L}^{2}(\Omega)$ (see [2]). Then following Verfürth and Braess [13], one can obtain under a saturation assumption that:

$$
\left\|\mathbf{V}-\mathbf{V}_{h}\right\|\|+\| s-s_{h}\left\|_{0, \Omega} \leq c \widetilde{\eta}+\frac{c}{h}\right\| \mathbf{G}_{h}^{n-1} \|_{0, e}\left(\sum_{e \in \mathcal{E}_{h}} h_{e}^{-1} \eta_{e}^{2}\right)^{1 / 2}
$$

where $\widetilde{\eta}$ is defined by:

$$
\widetilde{\eta}=\left(\sum_{K \in \mathcal{T}_{h}} \eta_{K, 1}^{2}+\sum_{K \in \mathcal{T}_{h}} \eta_{K, 2}^{2}+\sum_{e \in \mathcal{E}_{h}} h_{e}^{-1} \eta_{e}^{2}\right)^{1 / 2} .
$$

A lower bound can also be obtained but is not optimal.

\subsection{Numerical results}

We consider the same case of oil production as the one presented in Section 4.3.

In Figure 14, we represent the local estimators $\eta_{K}, \eta_{K, 1}, \eta_{K, 2}$ and $\left(\sum_{e \in \partial K} h_{e}^{-1} \eta_{e}^{2}\right)^{1 / 2}$ calculated for different meshes $\mathcal{T}_{1}, \mathcal{T}_{2}$ and $\mathcal{T}_{3}$ introduced in Section 4.3, after a one-month production. The approximate pressure and temperature on the previous meshes were given in Figure 9. 


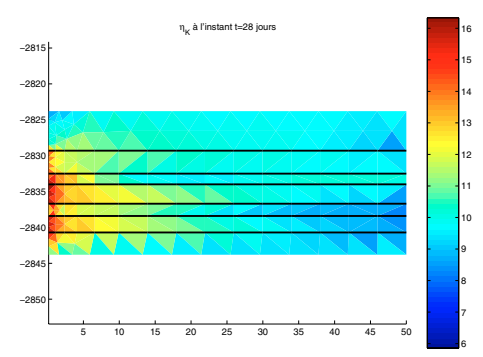

(a) $\eta_{K}-\operatorname{Mesh} \mathcal{T}_{1}$

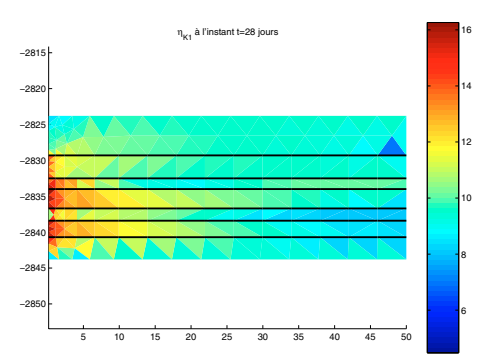

(d) $\eta_{K, 1}-\operatorname{Mesh} \mathcal{T}_{1}$

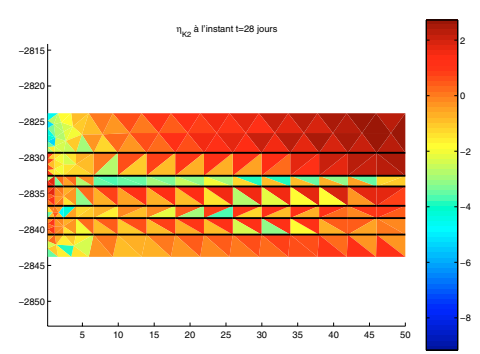

(g) $\eta_{K, 2}-\operatorname{Mesh} \mathcal{T}_{1}$

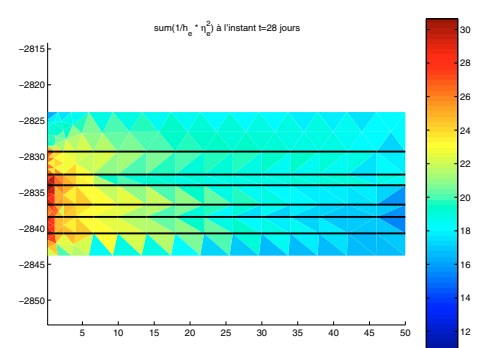

(j) $\left(\sum_{e \in \partial K} h_{e}^{-1} \eta_{e}^{2}\right)^{1 / 2}-$ Mesh $\mathcal{T}_{1}$

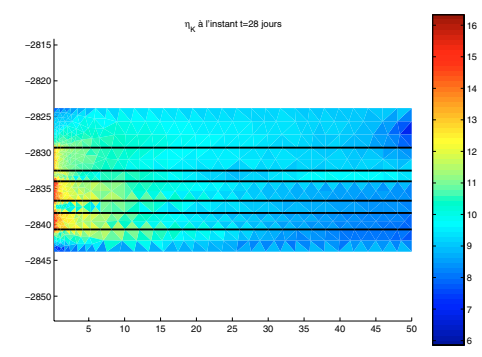

(b) $\eta_{K}-\operatorname{Mesh} \mathcal{T}_{2}$

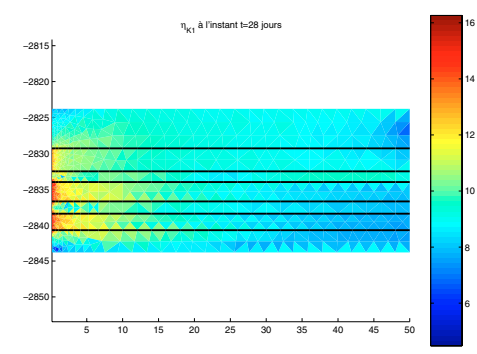

(e) $\eta_{K, 1}-\operatorname{Mesh} \mathcal{T}_{2}$

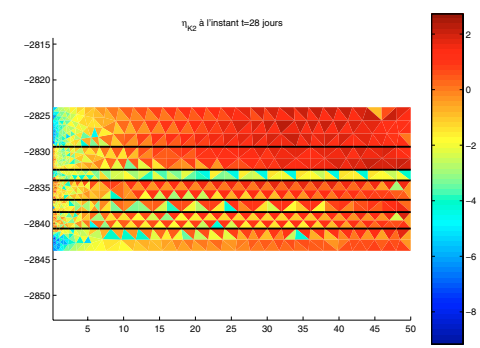

(h) $\eta_{K, 2}-$ Mesh $\mathcal{T}_{2}$

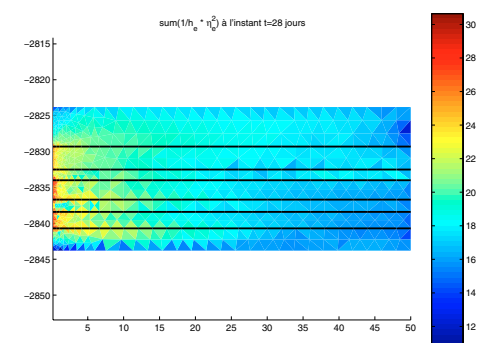

(k) $\left(\sum_{e \in \partial K} h_{e}^{-1} \eta_{e}^{2}\right)^{1 / 2}-$ Mesh $\mathcal{T}_{2}$

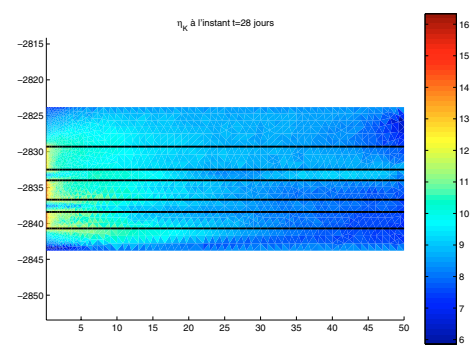

(c) $\eta_{K}-\operatorname{Mesh} \mathcal{T}_{3}$

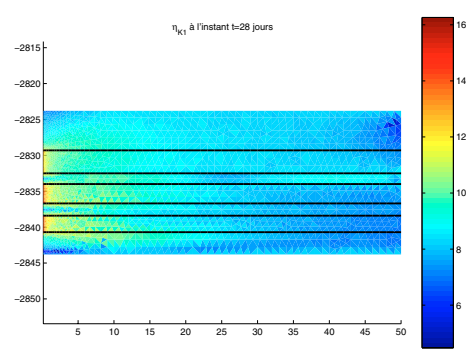

(f) $\eta_{K, 1}-$ Mesh $\mathcal{T}_{3}$

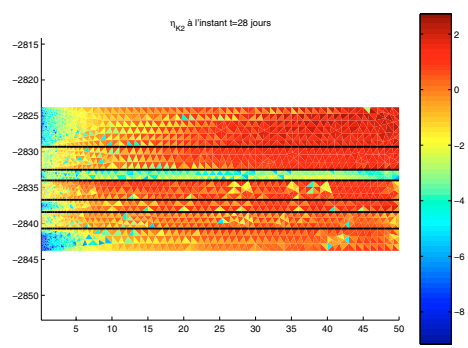

(i) $\eta_{K, 2}-$ Mesh $\mathcal{T}_{3}$

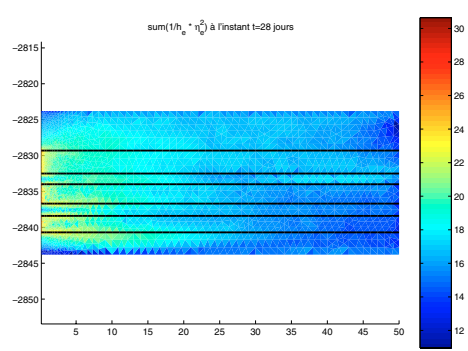

(l) $\left(\sum_{e \in \partial K} h_{e}^{-1} \eta_{e}^{2}\right)^{1 / 2}-$ Mesh $\mathcal{T}_{3}$

FIGURE 14. Local estimators for different mesh refinements.

As expected, the quality of the estimators increases with the mesh refinement. Moreover, one can notice that the area requiring a local refinement of the mesh is the one located near the wellbore, i.e. where the velocity is the highest. 


\section{REFERENCES}

[1] C. Abchir, Modélisation des écoulements dans les réservoirs souterrains avec prise en compte des interactions puits/réservoir. Thèse de doctorat, Université de Saint-Etienne (1992).

[2] M. Amara, D. Capatina, B. Denel and P. Terpolilli, Modelling, analysis and numerical approximation of flow with heat transfer in a petroleum reservoir, Preprint No. 0415, Université de Pau (2004) (http://lma.univ-pau.fr/publis/publis.php).

[3] G. Bourdarot, Well testing: Interpretation methods. Editions Technip, Paris (1998).

[4] S. Brenner and R. Scott, The mathematical theory of Finite Element Methods. Springer Verlag, New York (1994).

[5] F. Brezzi and M. Fortin, Mixed and Hybrid Finite Element Methods. Springer Verlag, New York (1991).

[6] G. Chavent and J.E. Roberts, A unified physical presentation of mixed, mixed-hybrid finite elements and standard finite difference approximations for the determination of velocities in waterflow problems. Adv. Water Resources 14 (1991) 329-348.

[7] P.G. Ciarlet, The finite element method for elliptic problems error analysis. North Holland, Amsterdam (1978).

[8] R.E. Ewing, J. Wang and S.L. Weekes, On the simulation of multicomponent gas flow in porous media. Appl. Numer. Math. 31 (1999) 405-427.

[9] P. Grisvard, Elliptic problems on non-smooth domains. Pitman, Boston (1985).

[10] F. Maubeuge, M. Didek, E. Arquis, O. Bertrand and J.-P. Caltagirone, Mother: A model for interpreting thermometrics. SPE 28588 (1994).

[11] D.Y. Peng and D.B. Robinson, A new two-constant equation of state. Ind. Eng. Chem. Fundam. 15 (1976) 59-64.

[12] J.E. Roberts and J.-M. Thomas, Mixed and Hybrid Methods, in Handbook of Numerical Analysis Vol. II. North Holland, Amsterdam (1991) 523-639.

[13] R. Verfürth and D. Braess, A posteriori error estimator for the Raviart-Thomas element. SIAM J. Numer. Anal. 33 (1996) 2431-2444.

To access this journal online:

www.edpsciences.org 